\title{
The PdBI Arcsecond Whirlpool Survey (PAWS): The Role of Spiral Arms in Cloud and Star Formation
}

\author{
Eva Schinnerer ${ }^{1}$, Sharon E. Meidt ${ }^{1}$, Dario Colombo ${ }^{2}$, Rupali Chandar ${ }^{3}$, Clare L. Dobbs ${ }^{4}$, Santiago García-Burillo ${ }^{5}$, Annie Hughes ${ }^{6}$, \\ Adam K. Leroy ${ }^{7}$, Jérôme Pety ${ }^{8,9}$, Miguel Querejeta ${ }^{1}$, Carsten Kramer ${ }^{10}$, and Karl F. Schuster ${ }^{9}$ \\ MPI for Astronomy, Königstuhl 17, D-69117, Heidelberg, Germany \\ ${ }^{2}$ MPI for Radioastronomy, Auf dem Hgel, Bonn, Germany \\ ${ }^{3}$ Department of Physics and Astronomy, The University of Toledo, RO 106, Toledo, OH 43606, USA \\ ${ }^{4}$ School of Physics and Astronomy, University of Exeter, Stocker Road, Exeter EX4 4QL, UK \\ ${ }^{5}$ Observatorio Astronómico Nacional-OAN, Observatorio de Madrid Alfonso XII, 3, E-28014, Madrid, Spain \\ ${ }^{6}$ IRAP, 9, avenue du Colonel Roche, BP 44346-31028 Toulouse cedex 4, France \\ ${ }^{7}$ Department of Astronomy, The Ohio State University, 140 W. 18th Avenue, Columbus, OH 43210, USA \\ ${ }^{8}$ Institut de Radioastronomie Millimétrique, 300 Rue de la Piscine, F-38406, Saint Martin d'Hères, France \\ ${ }^{9}$ Observatoire de Paris, 61 Avenue de 1'Observatoire, F-75014, Paris, France \\ ${ }^{10}$ Instituto Radioastronomía Milimétrica, Av. Divina Pastora 7, Nucleo Central, E-18012, Granada, Spain \\ Received 2016 August 13; revised 2016 December 2; accepted 2017 January 7; published 2017 February 8
}

\begin{abstract}
The process that leads to the formation of the bright star-forming sites observed along prominent spiral arms remains elusive. We present results of a multi-wavelength study of a spiral arm segment in the nearby grand-design spiral galaxy M51 that belongs to a spiral density wave and exhibits nine gas spurs. The combined observations of the (ionized, atomic, molecular, dusty) interstellar medium with star formation tracers (H II regions, young $<10$ Myr stellar clusters) suggest (1) no variation in giant molecular cloud (GMC) properties between arm and gas spurs, (2) gas spurs and extinction feathers arising from the same structure with a close spatial relation between gas spurs and ongoing/recent star formation (despite higher gas surface densities in the spiral arm), (3) no trend in star formation age either along the arm or along a spur, (4) evidence for strong star formation feedback in gas spurs, (5) tentative evidence for star formation triggered by stellar feedback for one spur, and (6) GMC associations being not special entities but the result of blending of gas arm/spur cross sections in lower resolution observations. We conclude that there is no evidence for a coherent star formation onset mechanism that can be solely associated with the presence of the spiral density wave. This suggests that other (more localized) mechanisms are important to delay star formation such that it occurs in spurs. The evidence of star formation proceeding over several million years within individual spurs implies that the mechanism that leads to star formation acts or is sustained over a longer timescale.
\end{abstract}

Key words: galaxies: individual (M51a, NGC 5194) - galaxies: ISM

\section{Introduction}

The role and importance of spiral arms in the star formation process in galaxy disks is a long-standing issue. Early morphological studies already recognized different structures associated with pronounced spiral arms emanating, often perpendicularly, from the arm. These features are referred to as spurs if caused by luminous (stellar) overdensities, feathers when they are due to absorption features, and pearls consisting of $\mathrm{H}$ II regions (for a full account of the history and nomenclature, see, e.g., the introduction of La Vigne et al. 2006). In particular, spurs seen as enhancements in (blue) optical light have long been recognized as special locations for the formation of stars in these galaxies (e.g., Elmegreen 1980). Corder et al. (2008) presented the first detections of molecular gas line emission coincident with such spurs in a region of the nearby spiral galaxy M51. This strongly suggests that these spurs have counterparts in molecular gas and thus a strong connection to the star formation process in spiral arms. Their analysis is still the only study focusing on the properties of gas spurs.

The last decade has seen substantial advances in studying the ISM in galaxies using numerical simulations. Recent research has demonstrated the importance of spiral shocks in the formation of giant molecular clouds (GMCs). In spiral shocks, collisions between clouds occur on relatively short timescales, allowing clouds to readily coalesce into GMCs (e.g., Tan 2000; Kim \& Ostriker 2002; Dobbs 2008). Gravitational interactions between clouds enhance this process, while the higher densities in spiral arms also facilitate gravitational instabilities (Shetty \& Ostriker 2006; Dobbs 2008). These numerical models now include heating and cooling of the ISM, self-gravity, and supernovae feedback (e.g., Dobbs et al. 2011). Furthermore the calculations have sufficient resolution to predict many properties of clouds, such as their masses and virial parameters (e.g., Dobbs 2008; Dobbs et al. 2011), as well as the time evolution of GMC and star formation (Dobbs \& Pringle 2013). The results of these simulations, however, need constraints from actual observations.

M51a a nearby ( $D \sim 7.6 \mathrm{Mpc}$; Ciardullo et al. 2002), almost face-on $\left(i \sim 22^{\circ}\right.$; Colombo et al. 2014b) disk galaxy with a clear spiral pattern, provides an excellent test bed for theoretical models and to study the link between spiral structure and star formation. The close relation between large complexes of GMCs and star formation sites have been noted already by Vogel et al. (1988), and also pointed out for clusters of stellar clusters by Bastian et al. (2005). The inner spiral arm pattern in M51 is very likely caused by a density wave, which is present as perturbation to the gravitational potential of the disk (e.g., Tully 1974; Elmegreen et al. 1989; Vogel 
et al. 1993; Meidt et al. 2008; Colombo et al. 2014b) and is thus ideal for a detailed study of the properties of the interstellar medium (ISM) and star formation across a spiral arm. M51 has been observed at essentially all wavelengths and is one of the best-studied grand-design spiral galaxies in the nearby universe. Recently, high spatial resolution observations of the ISM and star formation activity have been assembled and homogenized for the PdBI Arcsecond Whirlpool Survey (PAWS) database (Schinnerer et al. 2013). For our analysis, we make use of this database.

The paper is organized as follows. First we briefly describe the data used in Section 2. The molecular gas and star formation properties across a spiral arm segment are determined in Section 3, while we relate these findings to the star formation process in Section 4. Implications of our findings for the general picture of star formation in spiral arms are discussed in Section 5. We summarize our results and conclude in Section 6.

\section{Data}

For our analysis we use the $\mathrm{CO}(1-0)$ data products from the PdBI Arcsecond Whirlpool Survey (PAWS; Schinnerer et al. 2013), tracing the bulk molecular gas in conjunction with ancillary data probing the other ISM phases as well as different stages of star formation activity.

The molecular gas distribution in the central $\sim 9 \mathrm{kpc}$ of M51 was obtained as part of the PAWS project (Schinnerer et al. 2013). This IRAM Large Program observed 60 pointings with the Plateau de Bure interferometer (PdBI) in all configurations from 2009 August to 2010 March and mapped the full galaxy system with the $30 \mathrm{~m}$ single dish telescope in 2010 May in the ${ }^{12} \mathrm{CO}(1-0)$ line. The resulting data cube (with short spacing correction) has a resolution of 1 !" $16 \times 0$ !" 97 (PA $73^{\circ}$ ), with an rms of $0.4 \mathrm{~K}$ per $5 \mathrm{~km} \mathrm{~s}^{-1}$ wide channel. In addition, data cubes at $3^{\prime \prime}$ and $6^{\prime \prime}$ resolution were obtained in a similar fashion, but using a different weighting of the $u v$ data. A detailed description of the data reduction is presented by Pety et al. (2013). We also use the moment maps derived from this PAWS data cube (for details, see Pety et al. 2013; Colombo et al. 2014b).

The ancillary data used and their potential modifications (i.e., astrometry) are described in detail in section Section 2 of Schinnerer et al. (2013). In short, we use the $24 \mu \mathrm{m}$ image from Spitzer processed with the HiRes algorithm (Dumas et al. 2011), the IRAC $8 \mu \mathrm{m}$ imaging from SINGS (Kennicutt et al. 2003) processed by the $S^{4} G$ data pipeline (Sheth et al. 2010) and corrected for stellar emission using the ICA method of Meidt et al. (2012), the HST I - H map (Schinnerer et al. 2013), and the HST ACS H $\alpha$ image from the legacy data set (Mutchler et al. 2005) with the prescription for continuum correction of Gutiérrez et al. (2011). In addition, the H I robust weighted data from the THINGS survey (Walter et al. 2008) and the Herschel PACS [C II] map is used (see Parkin et al. 2013; Schinnerer et al. 2013).

In addition, we use the catalog of 1507 GMCs identified in the PAWS area (Colombo et al. 2014a), the catalog of 7215 grouped H II regions (i.e., giant H II regions identified via a friends-of-friends algorithm; Lee et al. 2011), and the catalog of 3812 stellar clusters identified in and characterized by HST $U V B I+\mathrm{H} \alpha$ imaging (Chandar et al. 2016).

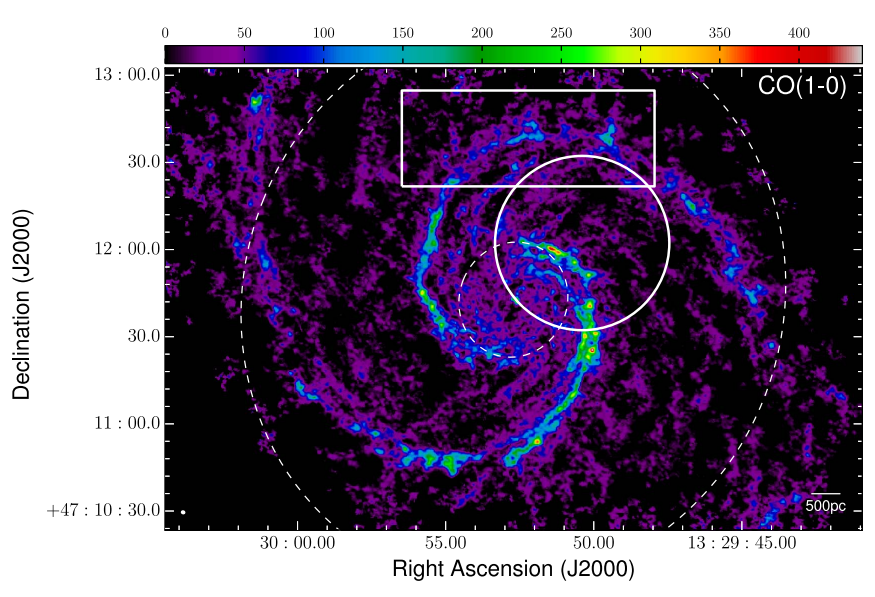

Figure 1. Molecular gas distribution as traced by $\mathrm{CO}(1-0)$ line emission in the central $9 \mathrm{kpc}$ of M51a, as observed by the PAWS project at $\sim 1^{\prime \prime}$ resolution. The region studied in detail here is indicated by a rectangular box, the pointing of Corder et al. (2008) by a circle. The two dashed circles centered on the nucleus of M51a are at the location of the co-rotation resonance of the nuclear bar (at $r=20^{\prime \prime}$ ) and the $m=2$ spiral mode (at $r=100^{\prime \prime}$; Meidt et al. 2008, 2013).

\section{Molecular Gas and Star Formation across a Spiral Arm in M51}

For the analysis in this paper we focus on a spiral arm region with a bona fide identification as a spiral density wave. ${ }^{11}$ Using an enhanced correction for dust emission in the Spitzer/IRAC $3.6 \mu \mathrm{m}$ image from the $S^{4} \mathrm{G}$ pipeline 5 (Querejeta et al. 2015), and considering information from beyond the edge of the PAWS FOV, Querejeta et al. (2016) move the co-rotation of the inner spiral pattern to $r \sim 100^{\prime \prime}$. This is also consistent with the kinematic decomposition of the line-of-sight velocity field traced by the cold ISM, as performed by Colombo et al. (2014b). That analysis clearly shows that the inner spiral pattern has properties consistent with a spiral density wave and reveals the kinematic signature of an additional, unique $m=3$ mode in the region of $20^{\prime \prime}<r<45^{\prime \prime}$; beyond $r \sim 100^{\prime \prime}$ analysis suggests that the spiral arm is more consistent with a material arm.

As Colombo et al. (2014b) provide convincing evidence that the superposition of the kinematically confirmed $m=3$ mode causes an enhancement in the potential of the southern arm and the southern arm bifurcates twice at $r \sim 45^{\prime \prime}$ and $r \sim 65^{\prime \prime}$, we restrict our analysis to $45^{\prime \prime}<r<75^{\prime \prime}$ of the northern arm only. This should also allow for more easy comparison to model predictions. The location of the area of interest within the PAWS region is presented in Figure 1.

\subsection{Molecular Gas Properties}

We utilize the PAWS $\mathrm{CO}(1-0)$ moment maps (Pety et al. 2013; Colombo et al. 2014b) and GMC catalog (Colombo et al. 2014a) to study the average properties of the molecular gas (see Section 3.1.1) and the GMCs (see Section 3.1.2) in different zones of our selected spiral arm region. We remind the reader that M51's cold ISM is predominantly molecular (Schuster et al. 2007), especially in the region under study. Recent studies of the dust-to-gas ratio in M51 (Mentuch Cooper et al. 2012) and of the conversion factor between CO

\footnotetext{
${ }^{11}$ Note that Corder et al. (2008) studied a region with zero torque associated with the circum-nuclear starburst ring; thus their studied features may have a different formation mechanism than the ones discussed here (see Figure 1).
} 

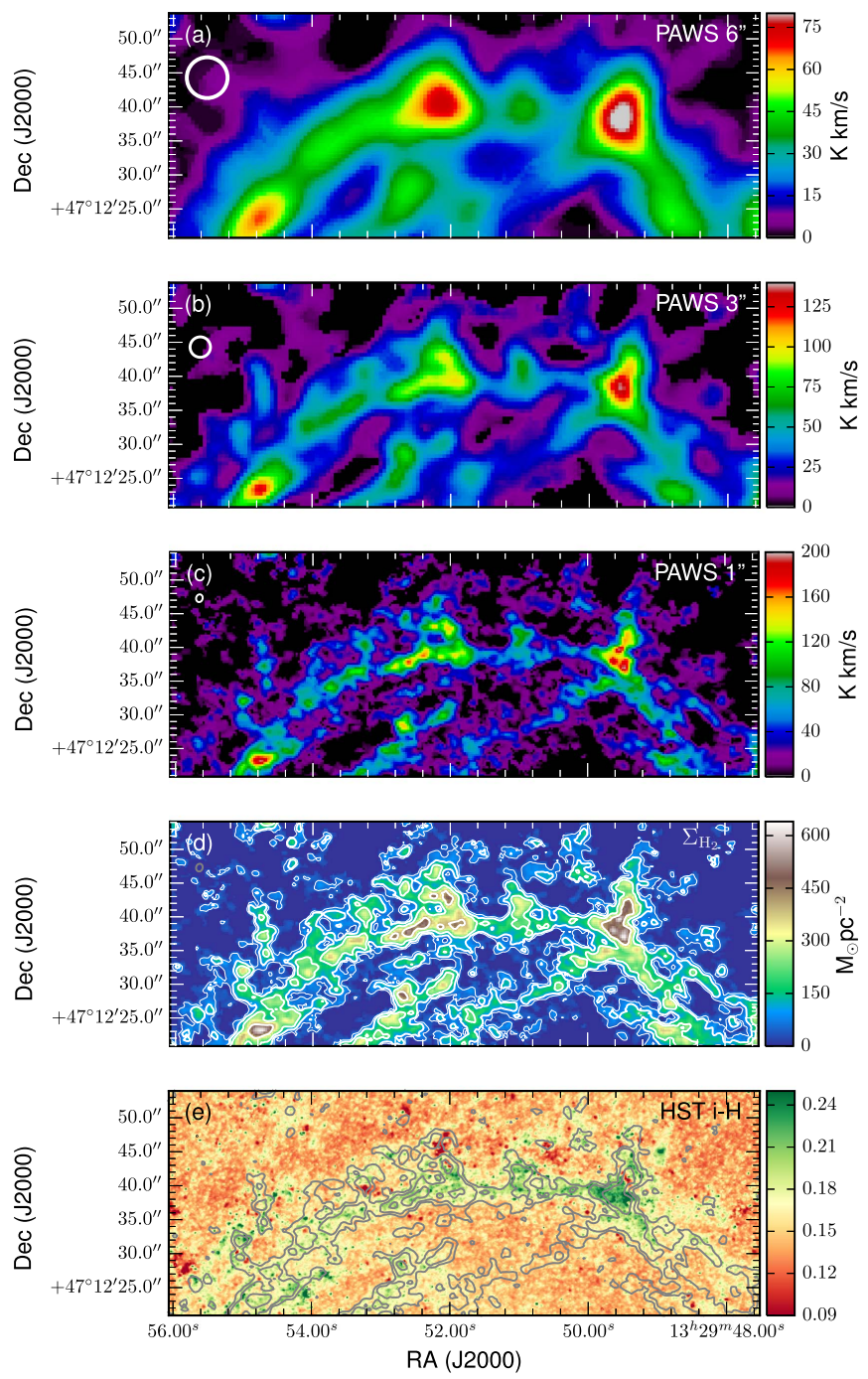

Figure 2. Molecular gas distribution as traced by $\mathrm{CO}(1-0)$ emission in the northern spiral arm of M51a as seen by PAWS, shown as follows. (a) ${ }^{12} \mathrm{CO}(1-0)$ intensity distribution at $6^{\prime \prime}$ resolution, (b) ${ }^{12} \mathrm{CO}(1-0)$ intensity distribution at $3^{\prime \prime}$ resolution, (c) ${ }^{12} \mathrm{CO}(1-0)$ intensity distribution at $\sim 1^{\prime \prime}$ native resolution, (d) surface density distribution of the molecular gas $\Sigma_{M_{\mathrm{H}_{2}}}$ with contours of 50 (thin line), 100, 200, and $400 M_{\odot} \mathrm{pc}^{-2}$ (thick lines), and (e) HST $i-H$ color map (green corresponding to the reddest colors) overlaid with gas surface density $\Sigma_{M_{\mathrm{H}_{2}}}$ contours from panel (d).

(1-0) line intensity and $\mathrm{H}_{2}$ gas mass comparing three independent methods (B. Groves et al. 2017, in preparation) show small variations similar to the uncertainties for SFR tracers at their spatial scales, considered ranging from $\mathrm{kpc}$ to $\mathrm{GMC}$ scale. Note that variations in the conversion between $\mathrm{CO}$ luminosity and molecular gas mass can be much larger when small regions within individual GMCs are considered.

In order to assess how the distribution/appearance of the molecular gas emission changes as a function of angular resolution (Figure 2), we compare the molecular gas maps as traced by $\mathrm{CO}(1-0)$ emission from $6^{\prime \prime}$ to $1^{\prime \prime}$ angular resolution. The CO(1-0) emission in the spiral arm segment shows five clear peaks with roughly equidistant spacing when mapped at $6^{\prime \prime}$ resolution. However, with increasing angular resolution these peaks are resolved into nine gas spurs that emanate almost perpendicular from the spiral arm. Comparison to the $H S T I-H$ color map reveals an excellent coincidence between the molecular gas spurs and the extinction feathers (i.e., the

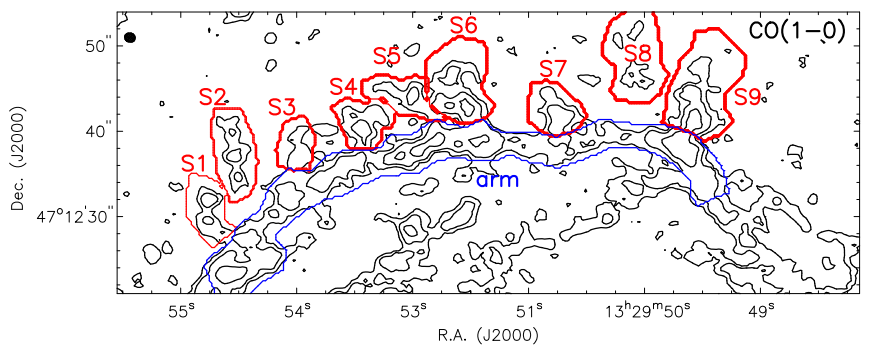

Figure 3. Nomenclature of identified gas spurs (red labels) and corresponding segments of the gas spurs (red contours), as well as the corresponding arm segment (the blue contour; see also Table 1).

extinction features leaving the main dust lane along the spiral arm). ${ }^{12}$ La Vigne et al. (2006) used HST ACS single band imaging to identify the extinction feathers in M51. All gas spurs in the spiral arm segment can be matched to their extinction feathers. This finding is similar to the result by Corder et al. (2008) for their spurs emanating from the inner part of the spiral arm that belongs to a kinematically different environment.

The good correspondence between the $\mathrm{CO}$ emission and the optical extinction as traced by the $H S T I-H$ image (Figure 2(d)) implies that no obvious spurs have been missed and that optical-near-IR color maps are a good predictor for the presence and location of gas spurs. This, in turn, means that feathers (i.e., elongated dust lanes) are caused by the presence of dense cold interstellar material and that the gas spurs and the extinction feathers belong to the same structures.

\subsubsection{Global Molecular Gas Properties in Arm and Spurs}

We identify nine spurs or spur-like features in the northern spiral arm segment (see Figure 3 for nomenclature); most of them have counterparts in optical extinction feathers as mapped by La Vigne et al. (2006). The exceptions are spurs S8, which does not connect to the arm, and S5, which La Vigne et al. (2006) associate with S6 as a single entity. Seven of the spurs are directly connected to the arm at our sensitivity limit, while almost all show distinct substructures (Figure 4(c)). The spacing between the bases of the spurs varies between $4^{\prime \prime}$ and $11^{\prime \prime}$ when deprojected (using an inclination of $22^{\circ}$ and position angle of $173^{\circ}$; see, e.g., Colombo et al. 2014b). The average spacing is $\sim 7 ! ! 5$. Their (deprojected) lengths are on average 6 ." 5, with the shortest spur S4 being about $25 \%$ shorter and the longest spur S9 being about $40 \%$ longer. The (deprojected) spurs have typical widths of $1^{\prime \prime}-2^{\prime \prime}(40-80 \mathrm{pc})$ in their thin structures and widen up to several arc-seconds in their thicker parts. The thicker parts are usually offset from the spiral arm. As the detected line emission is not smooth but exhibits emission peaks, this suggests internal structure within the molecular gas spurs. Defining regions for each spur and the arm (see red and blue contours in Figure 3), we measured global molecular gas properties such as integrated $\mathrm{CO}$ intensity, $\mathrm{H}_{2}$ gas mass $M_{\mathrm{H}_{2}}$, the corresponding $\mathrm{H}_{2}$ gas surface density $\Sigma_{M_{\mathrm{H}_{2}}}$, and the peak brightness temperature $T_{\mathrm{CO}}$ within each spur and the arm (see Table 1).

In most spurs the $\mathrm{CO}$ peak brightness temperature $T_{\mathrm{CO}}$ is typically as high or even higher in the spurs than the adjacent spiral arm segments (Figure 4(a)). However, the average (mean

\footnotetext{
12 For a summary of the naming conventions, we refer the reader to the introduction in La Vigne et al. (2006).
} 

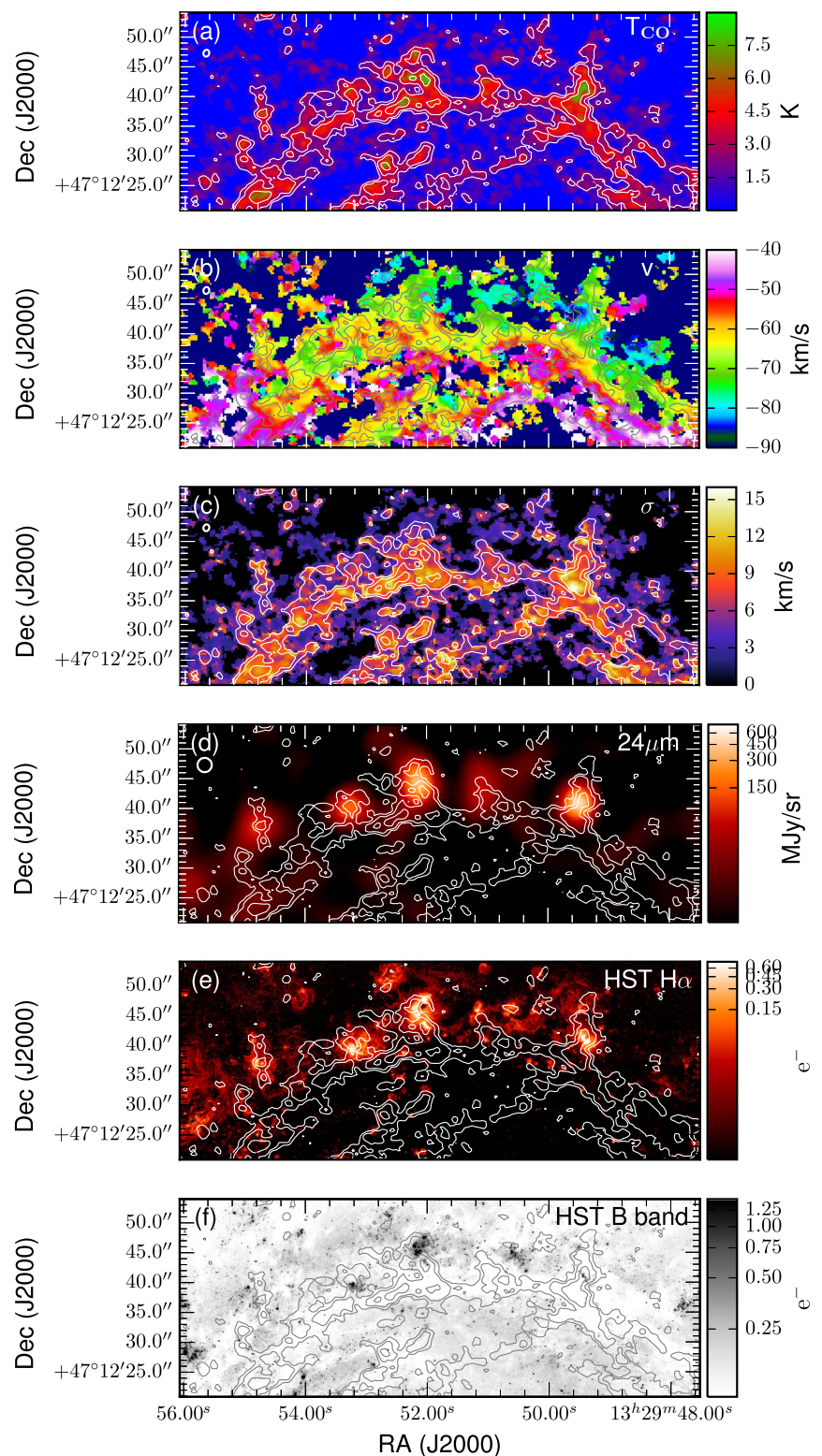

Figure 4. Properties of the molecular gas and distribution of star formation tracers in the northern spiral arm of M51a: (a) ${ }^{12} \mathrm{CO}(1-0)$ peak brightness temperature distribution, (b) ${ }^{12} \mathrm{CO}(1-0)$ velocity field colors ranging from -95 to $-45 \mathrm{~km} \mathrm{~s}^{-1}$, (c) ${ }^{12} \mathrm{CO}(1-0)$ velocity dispersion colors ranging from 0 to 16 $\mathrm{km} \mathrm{s}^{-1}$, (d) hot dust emission as traced by the MIPS $24 \mu \mathrm{m}$ continuum, (e) H II regions traced by the $H S T / \mathrm{ACS} \mathrm{H} \alpha$ emission, and (f) (young) stellar clusters as images in the $H S T /$ ACS $B$ band continuum. The contours in all images refer to the molecular gas surface density from Figure 2(d) (thick lines only).

or median) $T_{\mathrm{CO}}$ is generally about $20 \%-25 \%$ higher in the spiral arm $\left(\Delta T_{\mathrm{CO}, \text { arm }} \approx 3 \mathrm{~K}\right)$ than in the spurs (Table 1$)$. The exception is spur S6, which has an equally high mean value. Comparison of the mean and median $T_{\mathrm{CO}}$ shows that the mean is always larger than the median, with the exception of spur S3. While the difference is less than $\sim 5 \%$ for the arm and spurs S4 and S5, it is around $10 \%$ for the remaining spurs, except for spurs S3 and S9 where the difference is about 20\%. This suggests that the distribution of $T_{\mathrm{CO}}$ is skewed toward lower values in the majority of the spurs. It is interesting to note that the maximum $T_{\mathrm{CO}}$ value in two spurs $(\mathrm{S} 6, \mathrm{~S} 9)$ is higher than the maximum $T_{\mathrm{CO}}$ measured in the arm.
Using the integrated $\mathrm{CO}$ line emission, the picture reverses and the spiral arm becomes significantly brighter, also relative to most spurs (e.g., Figure 4(a)). Assuming the standard Galactic conversion factor between $\mathrm{CO}$ luminosity and molecular hydrogen mass of $\frac{N_{\mathrm{H}_{2}}}{I_{\mathrm{CO}}}=2 \times 10^{20} \mathrm{~cm}^{-2} \mathrm{~K}^{-1} \mathrm{~km}^{-1} \mathrm{~s}$, we calculated the distribution of the $\mathrm{H}_{2}$ surface density $\Sigma_{M_{\mathrm{H}_{2}}}$. In the spurs we find an average $\mathrm{H}_{2}$ gas surface density of $\Sigma_{M_{\mathrm{H}_{2}}} \approx 100 M_{\odot} \mathrm{pc}^{-2}$ (twice the value found by Corder et al. 2008 for their spurs), reaching maximum values of up to $400-500 M_{\odot} \mathrm{pc}^{-2}$ in the more prominent spurs (S6 and S9; see Table 1). However, we do not see a good correlation between the $\mathrm{H}_{2}$ surface density in the spurs and in the immediate adjacent spiral arm segments (Figure 2(d)), as $\Sigma_{M_{\mathrm{H}_{2}}}$ has both lower and higher values in the spurs than in the corresponding arm segment.

Taken together these results imply that although the gas surface density $\Sigma_{M_{\mathrm{H}_{2}}}$ is higher in the arm, the gas in the spurs is on average brighter based on the lower contrast between arm and spurs in the peak brightness temperature. This brightness increase could be caused by a higher filling factor, a higher gas volume density, or a higher gas kinematic temperature. From this finding (similar $T_{\mathrm{CO}}$, but different $\Sigma_{M_{\mathrm{H}}}$ ) immediately follows that the velocity dispersion $\sigma$ in the spurs is on average lower than $\sigma$ in the spiral arms (see Figure 4(d) and Table 1). The value for the spurs is on average a third lower than the arm value of $\sigma=8.1 \mathrm{~km} \mathrm{~s}^{-1}$. The typical gas mass within a spur is $M_{\mathrm{H}_{2}} \approx 4 \times 10^{6} M_{\odot}$, slightly higher than the amount of gas present in the spurs of Corder et al. (2008; when correcting for the different distance used). The spurs S6 and S9 contain about three times as much gas and both cover some of the largest area.

The spurs form a distinct kinematic environment, as the gas associated with the spurs shows strong deviations from the regular velocity field with velocity gradients (of roughly $5 \mathrm{~km} \mathrm{~s}^{-1}$ per arcsecond) along the minor axis of the spurs (e.g., S1, S3, S4, S6, and S9; see Figure 4(b)), though the direction of the gradient is not always the same. These strong streaming motions are not similar to the ones seen in the spiral arm itself, where the velocity gradient is generally largest across the arm width, and are almost perpendicular to the gradient seen across the spurs.

\subsubsection{Giant Molecular Cloud (GMC) Properties in Arm and Spurs}

In order to study the properties of GMCs in our defined spur and arm regions (see the red and blue contours in Figure 3), we utilize the PAWS GMC catalog (Colombo et al. 2014a) of GMCs identified via the CPROPS software (Rosolowsky \& Leroy 2006). In Figure 5(a) we highlight all GMCs associated with a spur (arm) as red (blue) circles. GMCs are mostly associated with the arm and the spurs; larger GMCs preferentially coincide with gas overdensities. A summary of the average properties of all GMCs found in either the arm or spur regions are listed in Table 2. (The individual properties of all identified GMCs are provided in Tables 5 and 6.)

The number of GMCs identified in the gas spurs ranges from 1 to 6 , with an average of 2.8 GMCs per spur. Spur GMCs have sizes of $\mathrm{r} \sim 50 \mathrm{pc}$ similar to the average arm GMC when the two large GMCs of spurs S4 and S8 are excluded. The typical line width of spur GMCs is slightly lower than for arm GMCs, consistent with the observed lower velocity dispersion in the spurs. The typical molecular gas mass of spur GMCs is about $3 \times 10^{6} M_{\odot}$, and therefore the typical spur GMC is about $25 \%$ 
Table 1

Molecular Gas Properties of the Northern Spiral Segment

\begin{tabular}{|c|c|c|c|c|c|c|c|c|c|c|c|}
\hline $\begin{array}{l}\text { Spur } \\
\#\end{array}$ & $\begin{array}{c}I_{\mathrm{CO}} \\
\left(\mathrm{K} \mathrm{km} \mathrm{s}^{-1}\right)\end{array}$ & $\begin{array}{c}S_{\mathrm{CO}} \\
\left(\mathrm{Jy} \mathrm{km} \mathrm{s}^{-1}\right)\end{array}$ & $\begin{array}{c}M_{\mathrm{H}_{2}} \\
\left(10^{6} M_{\odot}\right)\end{array}$ & $\begin{array}{c}\text { Area } \\
\left(10^{3} \mathrm{pc}^{2}\right)\end{array}$ & $\begin{array}{c}\overline{\sum_{M_{\mathrm{H}_{2}}}} \\
\left(M_{\odot} \mathrm{pc}^{-2}\right)\end{array}$ & $\begin{array}{c}\left\langle\Sigma_{M_{\mathrm{H}_{2}}}\right\rangle \\
\left(M_{\odot} \mathrm{pc}^{-2}\right)\end{array}$ & $\begin{array}{c}\Sigma_{M_{\mathrm{H}_{2}}}^{\max } \\
\left(M_{\odot} \mathrm{pc}^{-2}\right)\end{array}$ & $\begin{array}{l}\overline{T_{\mathrm{CO}}} \\
(\mathrm{K})\end{array}$ & $\begin{array}{c}\left\langle T_{\mathrm{CO}}\right\rangle \\
(\mathrm{K})\end{array}$ & $\begin{array}{c}T_{\mathrm{CO}}^{\max } \\
(\mathrm{K})\end{array}$ & $\left(\begin{array}{c}\bar{\sigma} \\
\left(\mathrm{km} \mathrm{s}^{-1}\right)\end{array}\right.$ \\
\hline 1 & 870 & 10.7 & 4.8 & 47.5 & 100 & 80 & 360 & 2.2 & 2.0 & 5.7 & 5.6 \\
\hline 2 & 930 & 11.3 & 5.1 & 63.9 & 80 & 60 & 290 & 2.1 & 1.7 & 6.1 & 4.9 \\
\hline 3 & 470 & 5.8 & 2.6 & 31.9 & 80 & 80 & 220 & 2.0 & 2.0 & 4.0 & 5.6 \\
\hline 4 & 920 & 11.2 & 5.1 & 43.1 & 120 & 110 & 300 & 2.7 & 2.6 & 5.9 & 5.8 \\
\hline 5 & 760 & 9.2 & 4.2 & 42.6 & 100 & 90 & 320 & 2.4 & 2.1 & 6.2 & 5.5 \\
\hline 6 & 2200 & 26.8 & 12.1 & 84.8 & 140 & 110 & 480 & 3.1 & 2.6 & 8.5 & 5.5 \\
\hline 7 & 980 & 11.9 & 5.4 & 44.5 & 120 & 110 & 320 & 2.7 & 2.5 & 6.2 & 5.7 \\
\hline 8 & 790 & 9.6 & 4.4 & 85.0 & 50 & 40 & 220 & 1.9 & 1.8 & 5.3 & 4.1 \\
\hline 9 & 2200 & 26.4 & 12.0 & 111.9 & 110 & 70 & 510 & 2.6 & 2.1 & 8.3 & 5.6 \\
\hline mean & 1120 & 13.7 & 6.2 & 61.7 & 100 & 80 & 340 & 2.4 & 2.2 & 6.2 & 5.4 \\
\hline arm & 15000 & 182 & 82.6 & 419.7 & 200 & 180 & 630 & 3.0 & 2.9 & 7.8 & 8.1 \\
\hline
\end{tabular}

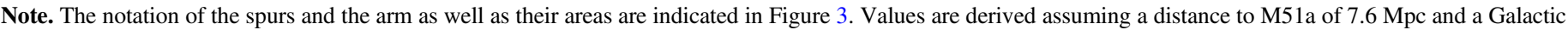

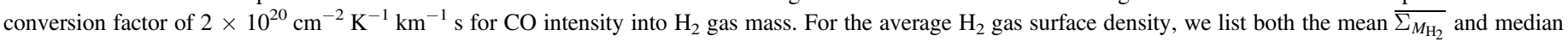
$\left\langle\Sigma_{M_{\mathrm{H}_{2}}}\right\rangle$ values. For the average $\mathrm{CO}$ peak brightness temperature, we list both the mean $\overline{T_{\mathrm{CO}}}$ and median $\left\langle T_{\mathrm{CO}}\right\rangle$ values.

more massive than the average arm GMC. The amount of molecular gas in GMCs per defined region (i.e., spur/arm) is still lower in the spurs than the arms; the difference is now only a factor of 1.5 times compared to two times for the gas surface density. Interestingly, the average fraction of gas mass in GMCs versus total gas mass per region is close to unity ( $\sim 95 \%$ ) for the spurs, ${ }^{13}$ while only about $\sim 75 \%$ of the molecular gas mass in the arm segment can be allocated to GMCs (see Table 2).

In summary, it appears that GMCs in the arm and spurs have fairly similar properties, with a slight preference for spur GMCs to be more massive. At the same time there is an indication that most molecular gas in the spurs is in GMCs, while a significant fraction of the gas in the arm might be distributed in less coherent (potentially more unbound) structures.

\subsection{Star Formation Properties}

The presence of massive star formation relative to the molecular gas distribution is investigated using three star formation rate tracers - namely hot dust, ionized gas, and blue optical light (see Section 3.2.1). A quantitative analysis of star formation in the different zones of the selected spiral arm region is made utilizing catalogs of (giant) $\mathrm{H}$ II regions (Lee et al. 2011) and young ( $\leqslant 10 \mathrm{Myr}$ ) stellar clusters (Chandar et al. 2016) identified in HST images (see Section 3.2.2).

\subsubsection{Massive Star Formation in Arm and Spurs}

The location of ongoing and recent massive star formation relative to the molecular gas arm and its spurs is shown in Figures 4(d)-(f). All three star formation tracers $(24 \mu \mathrm{m}$ emission from hot dust heated by massive stars, $\mathrm{H} \alpha$ emission from $\mathrm{H}$ II regions, and blue clusters of young stars in a HST B band image) show that (massive) star formation is almost exclusively associated with the gas spurs, and that no prominent star formation is taking place in the arm itself.

\footnotetext{
13 The reason that for some spurs more gas is found in GMCs compared to the spur area is due to the fact that the GMCs can extend beyond the spur area identified.
}

All five prominent $24 \mu \mathrm{m}$ emission peaks that can be identified in our selected region (Figure 4(d)) coincide with gas spurs (S2, S4, S6, S7, and S9), with the two brightest ones coinciding with spurs S6 and S9, which contain most of the molecular gas as traced by $\mathrm{CO}$ emission. The enhanced $24 \mu \mathrm{m}$ emission associated with the arm itself is very likely not due to embedded star formation but rather due to the higher gas density, as discussed by Schinnerer et al. (2013). Given the sensitivity of the data, there is no evidence for highly embedded star formation occurring in the arm. When using the full PAWS area and focusing on the brightest $24 \mu \mathrm{m}$ peaks (20 $\mathrm{MJy} \mathrm{sr}^{-1}<S_{24 \mu \mathrm{m}}<200 \mathrm{MJy} \mathrm{sr}^{-1}$ ), we find that indeed most peaks coincide with gas spurs. Excluding the central ringlike area, the numbers are 9/11 peaks in the southern arm and $7 / 9$ peaks in the northern arm.

The $\mathrm{H} \alpha$ emission (Figure 4(e)) is abundant north of the molecular gas arm and mainly arises from large (up to $5^{\prime \prime}$, i.e., $185 \mathrm{pc}$, diameter) shell-like structures, consistent with $\mathrm{H}$ II regions of ages of 5-10 Myr (Whitmore et al. 2011). All bright and large $\mathrm{H} \alpha$ emitting regions coincide with spurs (S2, S4, S6, and S9), while fainter emission is associated with three more spurs (S1, S7, and possibly S5). Interestingly, the location of the large $\mathrm{H} \alpha$ regions within the spurs varies from being close to the base of the spur (e.g., S3) to its tip (e.g., S6). Most of the gas arm itself is free of prominent $\mathrm{H} \alpha$ emission, except for the segments adjacent to spur S5 and S1. Over the entire PAWS area, basically all large H II region complexes are associated with gas spurs. We find, again excluding the central ring area, $11 / 12$ in the northern arm and 7/7 in the southern arm.

The HST $B$ band image shows the distribution of (young) stellar clusters relative to the molecular gas arm and its spurs (Figure 4(f)). Three prominent stellar cluster complexes are evident. Two of them coincide with spurs (S4, S6), while one lies off the arm roughly between spur S7 and S8. No prominent clusters are obvious within the gas arm, while most clusters are seen associated with spurs.

In summary, all three star formation rate tracers sensitive to the sequence from embedded star formation to stellar clusters devoid of their birth clouds are almost exclusively associated with gas spurs. This finding is not necessarily surprising, as Elmegreen (1980) previously noted that feathers (i.e., dust lanes corresponding to the gas spurs) are interspersed by pearls 

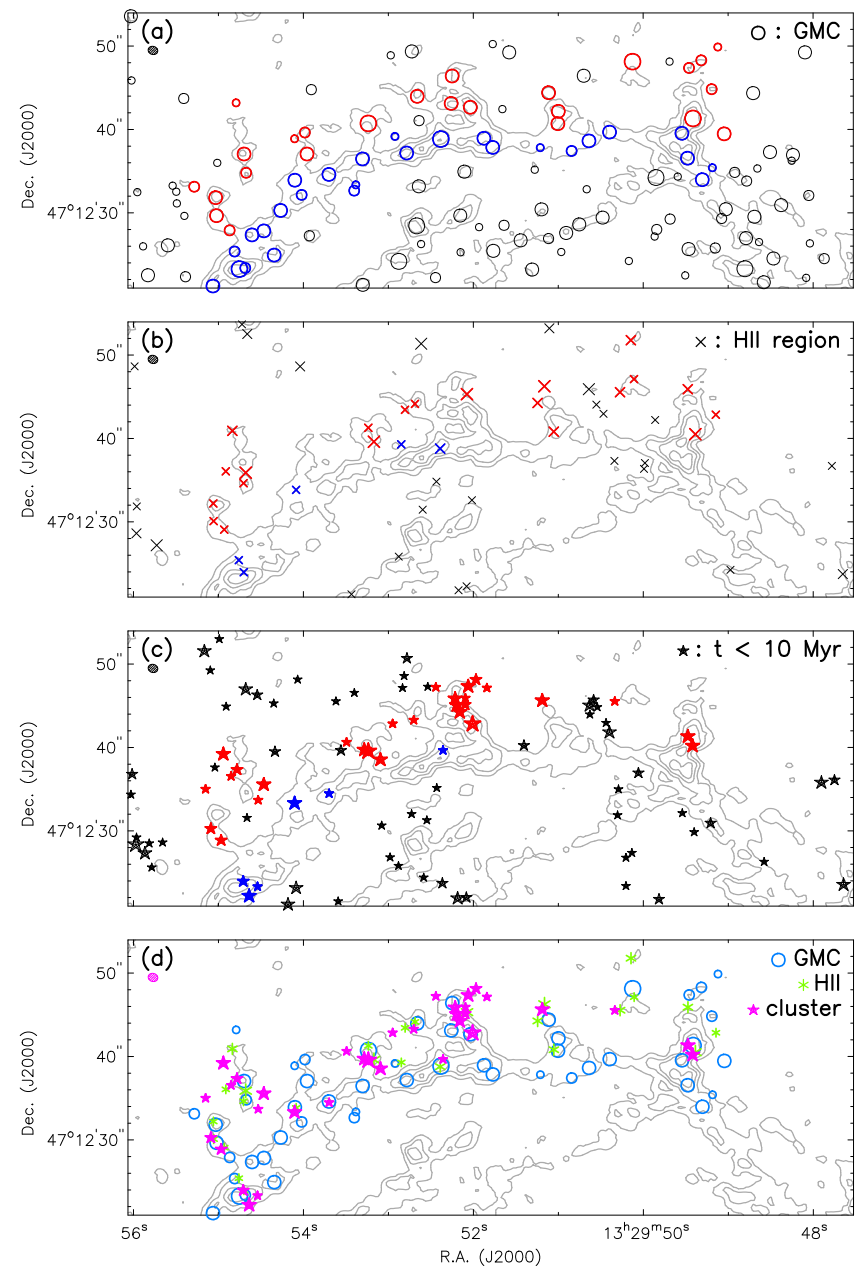

Figure 5. Location of identified GMCs (from Colombo et al. 2014a), H II regions (from Lee et al. 2011), and young stellar clusters (from Chandar et al. 2016) present in the region under study. Objects residing in our gas spiral arm (blue) and gas spurs (red) are color-coded: (a) GMCs from the catalog of Colombo et al. (2014a), where the size of the symbols corresponds to their respective mass (from small to large: $M_{\mathrm{GMC}}<5 \times 10^{5} M_{\odot}, 5 \times 10^{5} M_{\odot} \leqslant M_{\mathrm{GMC}}<1 \times 10^{6} M_{\odot}$, $1 \times 10^{6} M_{\odot} \leqslant M_{\mathrm{GMC}}<5 \times 10^{6} M_{\odot}, M_{\mathrm{GMC}}>5 \times 10^{6} M_{\odot}$ ); (b) H II regions from the catalog of Lee et al. (2011), where the size of the symbols corresponds to their respective $\mathrm{H} \alpha$ luminosity (from small to $\operatorname{large:} \log \left(L_{\mathrm{H} \alpha}\right)<37.5,37.5 \leqslant \log$ $\left.\left(L_{\mathrm{H} \alpha}\right)<39.0, \log \left(L_{\mathrm{H} \alpha}\right) \geqslant 39.0\right)$; and (c) stellar clusters younger than $10 \mathrm{Myr}$ from the catalog of Chandar et al. (2016), where the size of the symbols corresponds to their respective stellar mass (from small to large: $M_{\star}<5 \times 10^{3} M_{\odot}, 5 \times 10^{3} M_{\odot} \leqslant M_{\star}<1 \times 10^{4} M_{\odot}, 1 \times 10^{4} M_{\odot} \leqslant M_{\star}<$ $\left.5 \times 10^{4} M_{\odot}, M_{\star}>5 \times 10^{4} M_{\odot}\right)$. The three catalogs are combined in the last panel (d).

(i.e., large $\mathrm{H}$ II regions). This strongly suggests that there might be a causal link between gas spurs and massive star formation. We find no evidence for prominent ongoing star formation in the molecular gas arm itself.

\subsubsection{H II Regions and Young ( $\leqslant 10$ Myr) Stellar Clusters in Arm and Spurs}

We utilize the grouped H II region catalog of Lee et al. (2011) that is based on the HST Heritage Program imaging of M51. The authors have corrected the $\mathrm{H} \alpha$ flux for [N II] contamination, Galactic foreground, and an intrinsic mean attenuation of $A_{\mathrm{V}} \approx 3.1$ (Scoville et al. 2001). ${ }^{14}$ In addition,

\footnotetext{
${ }^{14}$ We corrected the catalog entries to our adopted distance.
}

they identified giant and super-giant $\mathrm{H}$ II regions via a friendsof-friends algorithm. We associate an $\mathrm{H}$ II region (of $\log \left(L_{\mathrm{H} \alpha}\left(\mathrm{erg} \mathrm{s}^{-1}\right) \geqslant 37.0\right)$ with a spur or the arm if its center falls within our defined regions. The average $\mathrm{H}$ II region properties are summarized in Table 3, while Table 7 lists all the associated H II regions from the catalog of Lee et al. (2011), corrected to our adopted distance and astrometry (for details on the astrometry, see Schinnerer et al. 2013).

As expected from the distribution of the $\mathrm{H} \alpha$ line emission, most $(21 / 26)$ of the grouped H II regions of Lee et al. (2011) belong to spurs (see Figure 5(b) and Table 3), despite the fact that the spurs and the arm encompass roughly similar areas. On average the $\mathrm{H}$ II regions in the spurs are twice as large, with a radius of $r \approx 70 \mathrm{pc}$, and over one order of magnitude brighter $\left(L_{\mathrm{H} \alpha} \approx 6 \times 10^{38} \mathrm{erg} \mathrm{s}^{-1}\right)$ than the $\mathrm{H}$ II regions in the arm. Consequently, the $\mathrm{H} \alpha$ luminosity normalized by the area in the spurs is about 30 times higher than in the arm. Of the four super-giant $\mathrm{H}$ II regions with $\log \left(L_{\mathrm{H} \alpha}\left(\mathrm{erg} \mathrm{s}^{-1}\right)\right)>39.0$, similar to 30 Doradus in the LMC or NGC 604 in M33, present in the area under investigation, three reside in spurs (S4, S6, S9), while the remaining one is located north of spur S9. Of the nine $\mathrm{H}$ II regions with $37.5 \leqslant \log \left(L_{\mathrm{H} \alpha}\left(\mathrm{erg} \mathrm{s}^{-1}\right)\right)<39.0$ present in our defined region, eight are located in spurs and one in the arm (next to spur S5 in the middle of the spiral arm), while several more are found north of the arm and spurs, with a typical distance of about $10^{\prime \prime}$. It is interesting to note that one spur (S3) does not contain any $\mathrm{H}$ II regions.

About 3500 stellar clusters with a $90 \%$ completeness level down to $m_{V} \approx 23$ have been identified in a summed HST BVI image by applying certain selection criteria on morphology (Chandar et al. 2011, 2016). The ages and masses are estimated using $U, B, V, I$, and (non-continuum-subtracted) $\mathrm{H} \alpha$ aperture photometry, together with population synthesis models (for a more detailed discussion of the young stellar clusters, see Calzetti et al. 2010). We select all stellar clusters with derived ages of $t \leqslant 10 \mathrm{Myr}$ from the catalog that are located in our area of interest. Again, we associate these young stellar clusters with spurs and the arm if their position falls within our defined regions. The average properties of these clusters are summarized in Table 4, while the individual cluster properties are listed in Table 8. All values have been corrected to our adopted distance.

We associate a total of 28 young stellar clusters with the spurs (see Figure 5(c)). One-third is found in spur S6 alone, one-sixth in spur S2 and S4 each, none in spur S3, and the remaining third is distributed in the other five spurs. A group of six stellar clusters is located between the two spurs S7 and S8, about $4^{\prime \prime}$ north of the gas arm. The six stellar clusters found in the arm are co-spatial with the $\mathrm{H}$ II regions and next to spurs S1, S3, and S5. There is a preference for the more massive stellar clusters to be associated with the spurs. The stellar clusters within the spurs are not uniformly distributed but tend to cluster together. This behavior has already been noted by Bastian et al. (2005), who identified complexes of stellar clusters within M51. The stellar clusters associated with spurs S6 and S8 correspond to their complexes C2 and D2, which are both consistent with homogeneous young stellar populations. Based on the high derived star formation rate surface density, Bastian et al. (2005) classify C2 as a localized starburst. The difference in the spatial distribution of young stellar clusters between spurs and the arm is also evident in the amount of (young) stellar mass per area, where 
Table 2

Properties of GMCs Located in the Northern Spiral Segment

\begin{tabular}{|c|c|c|c|c|c|c|c|}
\hline \multirow{2}{*}{$\begin{array}{l}\text { Spur } \\
\text { ID }\end{array}$} & \multicolumn{7}{|c|}{ GMC } \\
\hline & $\#$ & $\begin{array}{c}r \\
(\mathrm{pc})\end{array}$ & $\begin{array}{c}\Delta v \\
\left(\mathrm{~km} \mathrm{~s}^{-1}\right)\end{array}$ & $\begin{array}{c}M_{\mathrm{H}_{2}} \\
\left(10^{5} M_{\odot}\right)\end{array}$ & $\begin{array}{c}M_{\mathrm{H}_{2}} / A \\
\left(M_{\odot} / \mathrm{pc}^{2}\right)\end{array}$ & $\begin{array}{l}M_{\mathrm{H}_{2}, \mathrm{GMC}} \\
\left(10^{6} M_{\odot}\right)\end{array}$ & $\frac{M_{\mathrm{H}_{2}, \mathrm{GMC}}}{M_{\mathrm{H}_{2}, \text { tot }}}$ \\
\hline 1 & 4 & 38 & 7.8 & 11.0 & 93 & 4.4 & 0.92 \\
\hline 2 & 3 & 38 & 5.6 & 9.8 & 46 & 3.0 & 0.58 \\
\hline 3 & 3 & 48 & 5.8 & 10.3 & 97 & 3.1 & 1.19 \\
\hline 4 & 1 & 120 & 4.0 & 61.5 & 143 & 6.2 & 1.20 \\
\hline 5 & 1 & 60 & 4.4 & 29.9 & 69 & 3.0 & 0.71 \\
\hline 6 & 3 & 62 & 7.4 & 42.9 & 152 & 12.9 & 1.06 \\
\hline 7 & 3 & 55 & 4.9 & 25.6 & 173 & 7.7 & 1.42 \\
\hline 8 & 1 & 125 & 8.2 & 62.2 & 73 & 6.2 & 0.65 \\
\hline 9 & 6 & 45 & 5.6 & 14.5 & 78 & 8.7 & 0.73 \\
\hline mean & 2.8 & 66 & 6.0 & 29.7 & 103 & 6.1 & 0.94 \\
\hline arm & 27 & 51 & 7.2 & 23.9 & 150 & 60.1 & 0.73 \\
\hline
\end{tabular}

Note. The notation of spurs as provided in column (1) is indicated in Figure 3. The number of objects found is listed in column (2). The remaining columns give the mean value of the GMC radius (3), the line width (4), the $\mathrm{H}_{2}$ gas mass (5) derived from the $\mathrm{CO}(1-0)$ luminosity and corrected for He contribution as listed in the GMC catalog of Colombo et al. (2014a), the gas mass surface density per area analyzed (6), the total molecular gas mass in the GMCs (7) and compared to the total molecular gas mass (8) from Table 1. The properties of the individual GMCs listed in Table 5. The mean for all properties of GMCs found in the individual spurs is given in the second last row, and the corresponding properties for the arm segment are listed in the last row. Values are derived assuming a distance to M51a of $7.6 \mathrm{Mpc}$ and a Galactic conversion factor of $2 \times 10^{20} \mathrm{~cm}^{-2} \mathrm{~K}^{-1} \mathrm{~km}^{-1} \mathrm{~s}$ for $\mathrm{CO}$ intensity into $\mathrm{H}_{2}$ gas mass.

Table 3

Properties of H II Regions Located in the Northern Spiral Segment

\begin{tabular}{|c|c|c|c|c|}
\hline \multirow{2}{*}{$\begin{array}{l}\text { Spur } \\
\text { ID }\end{array}$} & \multicolumn{4}{|c|}{ H II Region } \\
\hline & \# & $\begin{array}{r}r \\
(\mathrm{pc})\end{array}$ & $\begin{array}{c}\log \left(L_{\mathrm{H} \alpha}\right) \\
\left(\log \left(\mathrm{erg} \mathrm{s}^{-1}\right)\right)\end{array}$ & $\begin{array}{c}\log \left(L_{\mathrm{H} \alpha}\right) / A \\
\left(\log \left(\mathrm{erg} \mathrm{s}^{-1} / \mathrm{pc}^{2}\right)\right)\end{array}$ \\
\hline 1 & 3 & 35 & 37.31 & 33.11 \\
\hline 2 & 4 & 50 & 38.00 & 33.80 \\
\hline 3 & 0 & $\ldots$ & $\ldots$ & $\ldots$ \\
\hline 4 & 2 & 102 & 38.89 & 34.55 \\
\hline 5 & 2 & 35 & 37.33 & 33.01 \\
\hline 6 & 1 & 188 & 39.47 & 34.54 \\
\hline 7 & 3 & 53 & 38.02 & 33.85 \\
\hline 8 & 3 & 40 & 37.55 & 33.10 \\
\hline 9 & 3 & 72 & 38.65 & 34.08 \\
\hline mean & 2.3 & 72 & 38.75 & 34.09 \\
\hline arm & 5 & 35 & 37.55 & 32.63 \\
\hline
\end{tabular}

Note. The notation of spurs as provided in column (1) is indicated in Figure 3. The number of objects found is listed in column (2). The remaining columns give the mean value of the $\mathrm{H}$ II region radius in column (3), the logarithm of the $\mathrm{H} \alpha$ luminosity $L_{\mathrm{H} \alpha}$ in column (4), and the $L_{\mathrm{H} \alpha}$ per area analyzed in column (5). The properties of the individual $\mathrm{H}$ II regions are listed in Table 7 and are taken from the group catalog of Lee et al. (2011). The mean for all properties of H II regions found in the individual spurs is given in the second to last row, and the corresponding properties for the arm segment are provided in the last row. Values are derived assuming a distance to M51a of $7.6 \mathrm{Mpc}$, and the properties of the $\mathrm{H}$ II regions have been corrected correspondingly.

the density is almost an order of magnitude higher for the spurs compared to the arm.

While the average age of the young stellar clusters in the spurs is about 50\% lower than in the arm, this is still within the uncertainty of a factor of 2 (Calzetti et al. 2010). However, it is interesting that the fraction of the youngest clusters with ages of $t<3 \mathrm{Myr}$ in the spurs (30\% or 9 out of 28) is about twice as
Table 4

Properties of Stellar Clusters Located in the Northern Spiral Segment

\begin{tabular}{lcccc}
\hline \hline \multirow{2}{*}{$\begin{array}{l}\text { Spur } \\
\text { ID }\end{array}$} & $\#$ & $\begin{array}{c}\log (t) \\
(\log (\text { year }))\end{array}$ & $\begin{array}{c}M_{\star} \\
\left(10^{4} M_{\odot}\right)\end{array}$ & $\begin{array}{c}M_{\star} / A \\
\left(M_{\odot} / \mathrm{pc}^{2}\right)\end{array}$ \\
\cline { 2 - 5 } & & 6.74 & 0.5 & 0.3 \\
\hline 1 & 3 & 6.60 & 1.5 & 1.2 \\
2 & 5 & $\ldots$ & $\ldots$ & $\ldots$ \\
3 & 0 & 6.54 & 1.8 & 2.1 \\
4 & 5 & 6.56 & 0.4 & 0.1 \\
5 & 1 & 6.42 & 2.0 & 2.4 \\
6 & 10 & 6.00 & 3.4 & 0.8 \\
7 & 1 & 6.78 & 0.1 & 0.02 \\
8 & 1 & 6.01 & 2.7 & 0.5 \\
9 & 2 & 6.46 & 1.5 & 0.9 \\
\hline mean & 3.1 & 6.64 & 0.7 & 0.02 \\
\hline arm & 6 & & & \\
\hline
\end{tabular}

Note. The notation of spurs as provided in column (1) is indicated in Figure 3. The number of young ( $\leqslant 10 \mathrm{Myr}$ stellar clusters found is listed in column (2). The remaining columns give the logarithm of the mean age in column (3), the mean stellar mass in column (4), and the mean stellar mass density (from clusters) in column (5). The properties of the individual stellar clusters are listed in Table 8 and are taken from the catalog of Chandar et al. (2016). The mean for all properties of young stellar clusters found in the individual spurs is given in the second last column, and the corresponding properties for the arm segment are listed in the last row. Values are derived assuming a distance to M51a of 7.6 Mpc, and the properties of the stellar clusters have been corrected correspondingly.

high as for the arm (17\% or 1 out of 6$)$. The young stellar clusters in the spurs are on average twice as massive compared to those in the arm, which is significant given the uncertainty on the stellar mass of $\approx 60 \%$ (Calzetti et al. 2010). Subsequently, the surface density of stellar mass contained in young stellar clusters is over four times higher in the spurs compared to the arm. As these systems should all have basically 
evacuated their surrounding birth material (i.e., the dust and gas that could potentially attenuate the stellar light), the difference between spurs and the arm cannot be explained by significantly higher extinction affecting the arm stellar clusters, unless one invokes a significantly different scale height for gas and dust in the arm compared to the spurs.

Similarly to the distribution of the SFR tracers (see Section 3.2.1), most of the $\mathrm{H}$ II regions and young stellar clusters are associated with the gas spurs. Interestingly, the four spurs that harbor the most luminous H II regions contain on average more massive young stellar clusters. The arm star formation sites are all close to spurs that only contain less luminous $\mathrm{H}$ II regions and less massive young stellar clusters (S1, S5), or even none at all (S3).

\section{The Star Formation Process in Gas Spurs}

\subsection{Relation of Spurs and Giant Molecular Cloud Associations (GMAs)}

The concept of Giant Molecular Associations (GMAs) was first introduced by Vogel et al. (1988) based on low-resolution interferometric observations of the molecular gas in the spiral arms of M51. These authors suggested that GMAs are formed out of GMCs that are already primed for star formation and that their formation is promoted by spiral density waves. A detailed followup study by Rand \& Kulkarni (1990) using similar CO data for most of the molecular gas disk in M51 found that 20 out of their 26 identified GMAs with molecular gas masses of (0.4-2.5) $\times 10^{7} M_{\odot}^{15}$ reside in the spiral arms and appear at their resolution of $10^{\prime \prime} \times 7^{\prime \prime}$ to be virially bound. In the literature it has become common to refer to coherent molecular gas structures above $10^{7} M_{\odot}$ as GMAs. For example, using their high-angular $4^{\prime \prime}$ resolution CO imaging of M51, Koda et al. (2009) identify several GMAs with molecular gas masses of $M_{\text {mol }}>10^{7} M_{\odot}$ associated with the spiral arm segment under study.

In order to assess the interpretation and identification of GMAs in M51, we use our findings from Section 3.1, where we investigated how the distribution/appearance of the molecular gas emission changes as a function of angular resolution (see Figure 2). The two most prominent emission peaks at $6^{\prime \prime}$ correspond to GMA A1 and A2 from Rand \& Kulkarni (1990) and can be associated with our spurs S5 plus S6 and S9, respectively. Inspecting the location of the 12 arm GMAs cataloged by Rand \& Kulkarni (1990) that fall within the full PAWS FOV, we find that 9 can clearly be associated with gas spurs (GMAs A1, A2, A3, A6, A8, A9, A10, A12, A13). One GMA coincides with a feather (La Vigne et al. 2006, though no clear gas spur is seen in this location that is close to the edge of the PAWS map). For GMA A4 it is hard to associate it with a clear spur/feather signature, as it is located in the arm region of the suppressed star formation (e.g., Meidt et al. 2013; Schinnerer et al. 2013), while GMA A11 falls into the interarm region. The mass of all GMCs associated with spurs S5 and S6 amounts to $1.6 \times 10^{7} M_{\odot}$ (i.e., close to the mass of GMA A1, the GMCs of spur S9 have a total of $8.7 \times 10^{6} M_{\odot}$ that corresponds to $\sim 40 \%$ of the molecular gas mass of GMA A2). As emission from the neighboring arm segment must have contributed to the total flux determined for the GMAs in the lower resolution imaging; this suggests that the GMAs are actually the blurred combination of

\footnotetext{
${ }^{15}$ Values corrected to our assumed distance of 7.6 $\mathrm{Mpc}$ and a Galactic conversion factor of $2 \times 10^{10} \mathrm{~cm}^{-2} \mathrm{~K}^{-1} \mathrm{~km}^{-1} \mathrm{~s}$.
}

gas in the spurs plus their neighboring arm segments (i.e., due to the low resolution, these "cross sections" appear bright).

Based on the previous analysis, we conclude that the GMAs identified by Rand \& Kulkarni (1990) in M51 are most likely an artifact of low-resolution observations where spatially separate emission from spurs and their neighboring arm are blended together. GMAs are therefore probably not single or special entities of multiple GMCs. This finding is not necessarily in disagreement with results of the high-angular resolution study of a segment of M51's southern spiral arm (Egusa et al. 2011), where the authors found a higher density of GMCs at the location of more massive GMAs identified by Koda et al. (2009) and interpreted this as evidence that GMAs are smooth structures that break up into collections of GMCs. As that high-resolution study missed $90 \%$ of the emission, it is difficult to link it directly to the fainter spurs that were identified in HST imaging (La Vigne et al. 2006).

\section{2. (Relative) Age of Star Formation Activity}

We use the spatial distribution of the products of star formation such as hot dust/PAH (via its $24 \mu \mathrm{m}$ and non-stellar $8 \mu \mathrm{m}$ emission), ionized and atomic hydrogen $(\mathrm{H} \alpha$ and $\mathrm{H} \mathrm{I}$ emission), as well as ionized carbon ([C II $] \lambda 158 \mu$ m emission) in conjunction with $\mathrm{H}$ II regions (including the morphology of the $\mathrm{H} \alpha$ emission) and young stellar clusters (see HST B band) relative to the distribution of the molecular gas (traced via $\mathrm{CO}$ ) (see e.g., Figure 6), to infer the relative age of the star formation activity associated with the spurs and arm, as well as the typical location of star formation sites relative to the arm. This information allows us to search for an age trend that would be expected if, for example, all star formation would be started at the same location (e.g., inside the arm). We note that the prominence (as traced by their brightness) of the star formation sites varies considerably across the spurs and could introduce some bias in our age assignment.

\subsubsection{Along the Spiral Arm}

As spur S3 contains abundant molecular gas but shows no sign for any associated star formation, nor any evidence of star formation impact, we consider it a potential site for future star formation (i.e., the relative "youngest" among our nine spurs).

Both spurs S7 and S9 have prominent $24 \mu \mathrm{m}$ and $\mathrm{H} \alpha$ emission with significantly fewer $B$ band clusters, suggesting that massive star formation has only recently started in these spurs. Spur S7 might be in a slightly earlier star formation phase than spur S9, given the faintness of the clusters in the $B$ band. This interpretation is consistent with the distribution of the ISM dissociation products (H I and [C II] line emission): an H I (but not [C II]) peak is associated with S7, while both emission lines are mainly found downstream of the major starforming site in $\mathrm{S} 9$ (i.e., peaks in $\mathrm{H} \alpha$ and hot dust/PAH emission). The most prominent, ongoing, massive star formation sites are associated with spurs S4 and S6, where all three star formation indicators are co-spatial with the $\mathrm{CO}$ emission. The difference in the [C II] over $\mathrm{H}$ I ratio between the two spurs could indicate that star formation might have been proceeding for a longer time in spur S6. It is also interesting to note that the spatial coincidence of $24 \mu \mathrm{m}, \mathrm{H} \alpha$ emission, and young stellar clusters implies that massive star formation has occurred within a spur over several Myr (e.g., spurs S2, S4, S6, S9). 


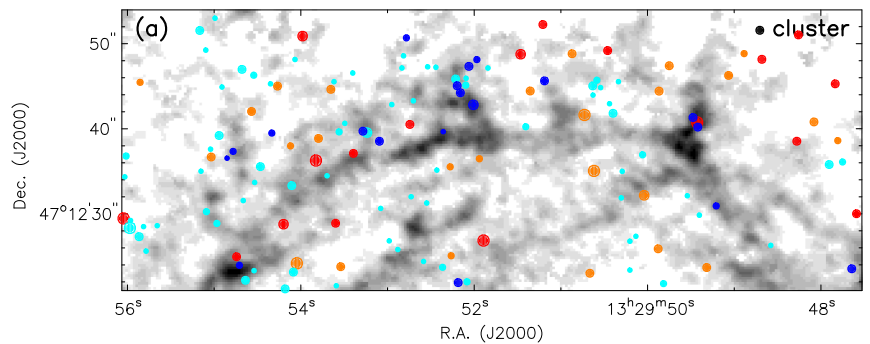

Figure 6. Detailed comparison of the location of stellar clusters (filled circles) relative to the molecular gas distribution (grayscale). The stellar clusters are shown by filled circles symbols (same size as for Figure 5(c)). The color coding corresponds to ages of $\log (t$ (year) $)<6.5$ (blue), $6.5 \leqslant \log (t$ (year) $)<7.0$ (cyan), $7.0 \leqslant \log (t$ (year) $)<8.2$ (orange), and $\log (t$ (year) $) \geqslant 8.2$ (red). It is noteworthy that clusters with ages of $\log (t$ (year) $)<6.5$ tend to be highly clusters, while the clustering is becoming less obvious for clusters with ages of $6.5 \leqslant \log (t$ (year) $)<7.0$ and is non-apparent for even older clusters.

Similar to the previous spurs, spur S2 also has all three star formation tracers associated with it; however, they are clearly spatially separated. The young clusters tend to be located on the edge of the gas spur, suggesting that star formation has been proceeding for a while already inside this spur. The situation in spur S1 is similar, although the $24 \mu \mathrm{m}$ emission is significantly reduced, implying that this star formation site is slightly older. The lack of associated $24 \mu \mathrm{m}$ emission together with significant $\mathrm{H} \alpha$ emission and young stellar clusters suggests that the formation of massive stars has just ceased in spur S5.

The over-density of stellar clusters that is located between the arm and spur S8 is the relative "oldest" star-forming site, as no $24 \mu \mathrm{m}$ emission is present and the $\mathrm{H} \alpha$ emission is very faint and diffuse, suggesting that the $\mathrm{H}$ II region has already dissolved. The presence of significant [C II] emission suggests that there is still a significant amount of ionizing photons from massive stars and/or that the recombination time for $\mathrm{C}^{+}$is much longer than for $\mathrm{H}^{+}$to $\mathrm{HI}$ to $\mathrm{H}_{2}$. Similarly, the distribution of the molecular gas as seen in the $\mathrm{CO}$ emission is more dispersed, suggesting that the previous massive star formation events have had a severe impact on the morphology and prominence of the spur. It also shows that a spur is not necessarily continuously fed by new material from the arm.

When using our crude classification for the age of the star formation site, we find no clear age trend across our nine spurs (color coding in Figure 7 from blue [youngest] to red [oldest]).

\subsubsection{Perpendicular to Spiral Arm = along a Spur}

The location of star formation sites relative to the spurs can be roughly classified into four categories: (a) in the arm next to a spur base, (b) at the base of the spur where it connects to the arm, (c) in the middle of the spur, and (d) at the tip of the spur. Spurs S1, S3, and S5 are next to star-forming sites in the arm, while spurs S4 and S9 have most star formation occurring at their base. Prominent star formation in the middle of the spur occurs in spur S1, S2, and the almost dispersed spur S8. In spurs S5, S6, and S7 most star formation is found at the tip of the gas spur. In short, no obvious trend of the star formation location along a spur is found among our nine spurs (see the open star symbols in Figure 7).

When we combine the age classification with the location of star formation along a spur, we find no preferred location for a given age, nor a trend along the spiral segment. However, it is interesting to note that some spurs (S6, S7, and S9) exhibit a clear age gradient along the spur, with more recent star

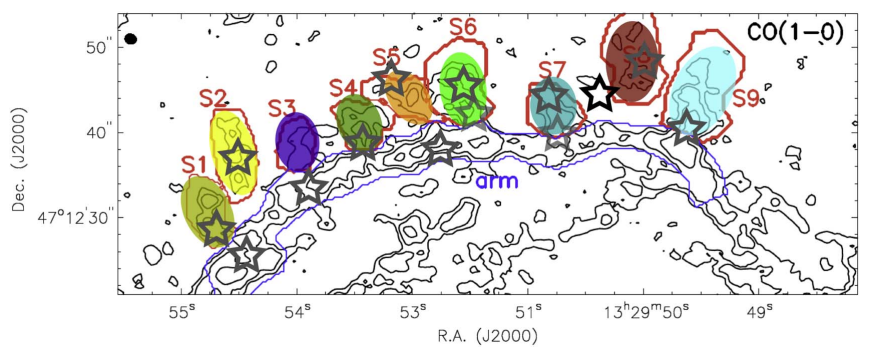

Figure 7. Relative age of star formation associated with our spurs (from young to old: dark blue-cyan-green-yellow-orange-dark red shading) based on different star formation tracers (see text for details). The preferred location of star-forming in each spur is marked by an open star symbol (dark gray). Star formation outside spur S8 (black open star) and in the arm close to spurs (light open star) is indicated as well. (See the text for details.)

formation activity being closest to the arm. For the remaining spurs, we see no clear spatial segregation between different tracers for star formation activity and impact.

Taken together this suggests that star formation is not started in a preferred, fixed location relative to the spiral arm (in each spur). However, there might be a preference for star formation onset more closely to the arm within each spur. As star formation typically proceeds for several Myr within an individual gas spur, this implies the onset mechanism has to act over a longer timescale or the star formation process itself is not instantaneous but can be sustained for a certain, few Myr long, time interval.

\subsection{Star Formation Feedback}

To study the impact of star formation on the ISM, we use the following data to investigate the dissociation of molecular hydrogen $(\mathrm{HI}$ and $\mathrm{H} \alpha$ ) and the $\mathrm{CO}$ molecule ([C II] line at $158 \mu \mathrm{m})$, as well as the heating of the interstellar dust $(8 \mu \mathrm{m}$ emission corrected for stellar contribution, and MIPS $24 \mu \mathrm{m}$ emission). Figure 8 shows a comparison of some of these tracers to the molecular gas distribution as seen via its $\mathrm{CO}$ emission. We discuss the geometry and properties of the individual spurs in this section.

General trends of the impact of star formation onto the ISM can be summarized as follows.

(i) The youngest stars heat the dust. Hot dust/PAH emission along the spurs is always coincident with molecular gas (emission from $\mathrm{CO}$; see Figure 8(d)), and the peaks in dust emission are consistent with the location of the youngest (i.e., below $3 \mathrm{Myr}$ ) stellar clusters. Due to the lower resolution of the dust emission of $\sim 2^{\prime \prime}$ compared to the HST imaging $(\sim 0$ "! 1$)$ for the young clusters, it is difficult to search for small but significant spatial offsets between the two tracers. However, in particular spurs S2 and S6 show some indication for an age differentiation, even among the youngest stellar clusters (or at least their impact onto the surrounding ISM).

(ii) The dissociation product of $\mathrm{CO}$, the $[\mathrm{C} \mathrm{II}]$ emission line, can be observed after $H$ II regions have ceased to exist. A prime example is spur S8, where a bright [C II] emission peak (Figure 8(c)) has no counterpart in $\mathrm{H} \alpha$ emission (Figure 8(a)). Interestingly, the brightest [C II] and $\mathrm{H} \alpha$ emission peaks (Figure 8(a)) do not show a 1-to-1 correspondence, and the same is true for [C II] and hot dust/PAH emission. This suggests that the [C II] emission in the spurs tends to reach its peak brightness at a 

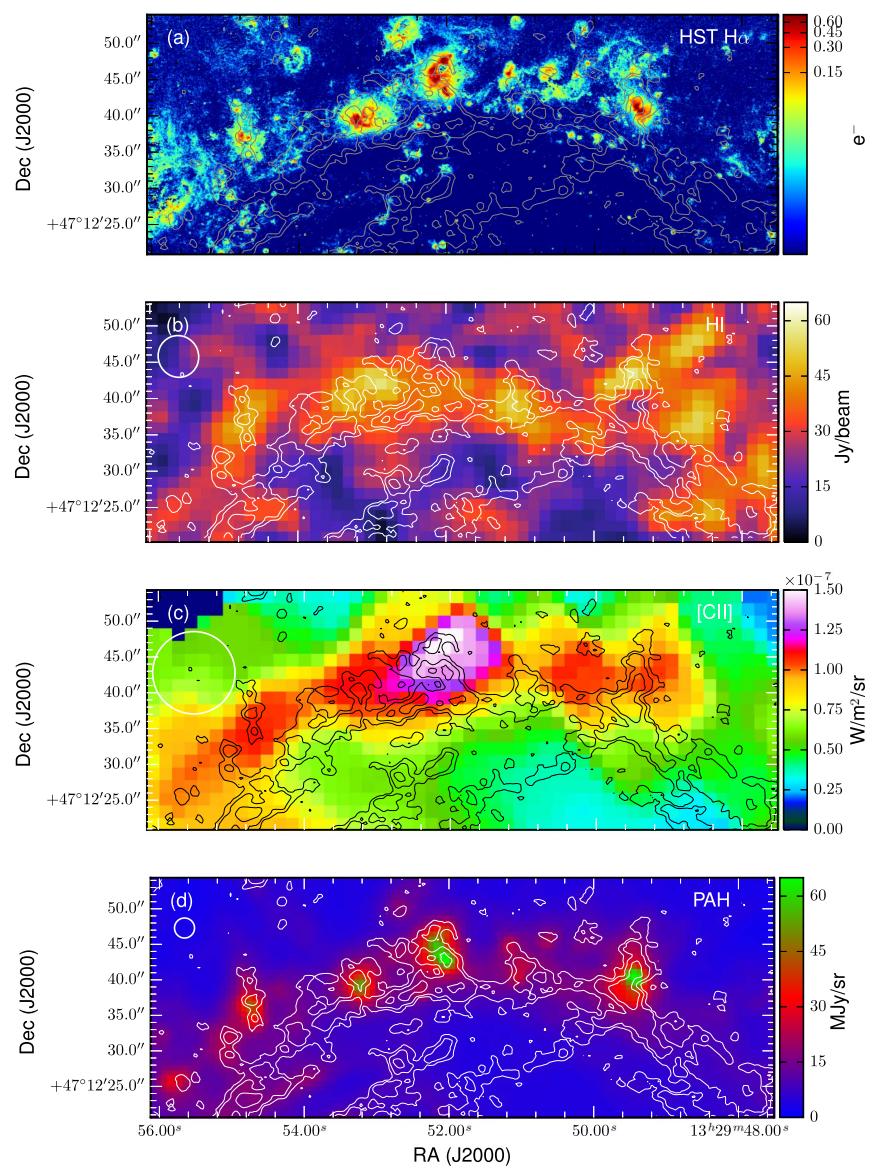

Figure 8. Properties of the molecular gas and distribution of ISM tracers in the northern spiral arm of M51a: (a) ionized hydrogen gas as traced by HST/ACS $\mathrm{H} \alpha$ emission, (b) atomic $\mathrm{H}$ I gas from VLA THINGS survey (Walter et al. 2008), (c) ionized carbon traced by [C II] line emission from PACS/ Herschel (e.g., Parkin et al. 2013), and (d) $8 \mu \mathrm{m}$ PAH emission from Spitzer IRAC imaging by SINGS (Kennicutt et al. 2003). The contours in all images refer to the molecular gas surface density from Figure 2(d) (thick lines only).

later point in time after the onset of star formation than the other two tracers and/or that [C II] emission has a longer decay time.

(iii) The observed $\mathrm{H}$ I line emission can be either an $\mathrm{H}_{2}$ dissociation or $\mathrm{H}^{+}$recombination product. Given the location of the HI peaks (Figure 8(b)), it seems that bright $\mathrm{HI}$ emission is more often observed as a recombination product (e.g., $\mathrm{H}$ I emission located downstream of spurs S5 and S8 with no associated H II emission) than a dissociation product (e.g., H I emission at base of spur S7 associated with $\mathrm{H}$ II emission and the connection to the spiral arm). However, it is clear that for our arm regions, elevated $\mathrm{H}$ I emission is never observed upstream of the spiral arm (i.e., south of the molecular gas arm). This is consistent with the very high molecular gas fraction of $>80 \%$ (Schuster et al. 2007) that makes the need for $\mathrm{H}_{2}$ formation out of atomic gas in/before the spiral arm shock obsolete. Note that Tilanus \& Allen (1989) already proposed that most of the H I emission associated with the inner spiral arms of M51 is due to H I dissociation based on the observed offset between the non-thermal radio continuum and the $\mathrm{HI}$ line emission. Our analysis suggests that the overall notion that $\mathrm{HI}$ emission is due to the star formation process downstream of the spiral arm is correct; however, the exact identification of $\mathrm{HI}$ as being an $\mathrm{H}_{2}$ dissociation or an $\mathrm{H}^{+}$recombination product is more subtle and will require more dedicated analysis.

In the following we summarize the properties of each spur. Spur S1-The combined light of the H II regions and young stellar clusters associated with spur S1 are the second faintest; their impact on the molecular ISM of the spur is not significant. The $\mathrm{H} \alpha, \mathrm{HI}$, and [C II] line emissions show no evidence for enhancement at the location of this spur. As the hot dust/PAH emission is slightly elevated, this suggests that some heating of the dust grains is happening.

Spur S2-Diffuse $\mathrm{H} \alpha$ emission is coinciding with the entire extent of the molecular gas spur S2. The brightest $\mathrm{H} \alpha$ emission is located slightly east of the middle peak of the molecular distribution. The hot dust/PAH emission is peaking between the two molecular gas emission peaks, just south of the $\mathrm{H} \alpha$ peak. The brightest $\mathrm{H}$ I emission in this spur is also found at the location of the hot dust/PAH peak. There is clearly enhanced [C II] emission associated with this spur; however, due to the low resolution of the data, it is difficult to draw any further conclusions.

Spur S3-No emission from $\mathrm{H} \alpha, \mathrm{H} \mathrm{I},[\mathrm{C} \mathrm{II}$ ], and/or $8 \mu \mathrm{m}$ $\mathrm{PAH} /$ hot dust is associated with spur S3. This implies that there is no evidence for star formation activity in the recent past ( $\lesssim 10-20$ Myr). This is consistent with the absence of $\mathrm{H} \mathrm{II}$ regions and young stellar clusters, and our interpretation of this spur as a potential site for future star formation.

Spur S4-The second brightest H II region (in our region under study) sits in spur S4. Its impact onto the surrounding ISM is obvious from enhanced H I, [C II], and hot dust/PAH emission arising from this spur. The diffuse $\mathrm{H} \alpha$ emission surrounds the base of the spur and extends through a significant fraction of the molecular spur toward its tip. It is interesting that a $\mathrm{H} \alpha$ shell fits nicely into the kink at the western part of the tip. The peak of the $\mathrm{H}$ I emission is offset from the prominent $\mathrm{H}$ II region toward the northwest and spur S5. This could indicate that hydrogen is already recombining again in this region, as only one young stellar cluster and no $\mathrm{HII}$ region is present in this area (see Figure 5). The hot dust/PAH emission coincides with the $\mathrm{H} \alpha$ emission, while the [C II] emission has no distinct peak.

Spur S5-The $\mathrm{H} \alpha$ emission in the tip of spur S5 has no prominent counterparts in the other tracers. This is not surprising, as both the $\mathrm{H}$ II regions and the young stellar cluster are the smallest of all the ones that are hosted in spurs. Thus no strong impact is necessarily expected. In addition, due to the proximity of the very prominent star formation in spur S6 and the low angular resolution of most tracers, it is difficult to uniquely associate them with spur S5.

Spur S6-The brightest H II region and the largest number of young stellar clusters (including the most massive one) are found in spur S6; thus a strong impact of the star formation onto the surrounding ISM is expected. A chain of $\mathrm{H}$ II regions is located along the hook-shaped gas spur. The regions in the south are surrounded by less diffuse $\mathrm{H} \alpha$ emission and are coincident with molecular gas and hot dust/PAH emission. The $\mathrm{H}$ II regions in the middle and the far north straddle the molecular gas emission peak of the spur. These regions are also accompanied with most of the young stellar clusters, while most of the older ( $3 \mathrm{Myr}<t \leqslant 10 \mathrm{Myr}$ ) clusters are found in the ridge between the molecular gas emission peaks at the base and the tip of the spur. The brightest peak in [C II] emission is 
associated with spur S6, and the peak within this brightest [C II] emission is found at the tip of the spur. Interestingly, no enhanced H I emission is seen from the tip of the spur, but it is rather seen from the base and the neighboring arm segment.

Spur S7-The situation for spur S7 is more complex; the H II region at the base of the spur is associated with a peak in $\mathrm{H} \mathrm{I}$ and $8 \mu \mathrm{m}$ hot dust/PAH emission, while the two $\mathrm{H}$ II regions at the tip of the spur are only co-spatial, with enhanced non-stellar $8 \mu \mathrm{m}$ emission. There is no direct evidence for [C II] emission arising from spur S7 at all, given the resolution of the data. This behavior could indicate that the impact of star formation is stronger close to the arm (given that the $\mathrm{H} \alpha$ luminosity of this region is only about $10 \%$ of the one from the regions at the tip) and/or that the $\mathrm{H}$ II regions at the tip are older, given their larger sizes.

Spur S8-The [C II] emission shows a clear peak located roughly between spur S8 and the neighboring arm segment. Interestingly, the young stellar clusters are on the eastern side of this peak, and no $\mathrm{H}$ II region is associated with this [C II] peak. However, faint, diffuse $\mathrm{H} \alpha$ emission is visible in the HST imaging of this region, while no $\mathrm{H}$ I peak is visible either. This suggests that the nearby young stellar clusters are providing enough energy input to dissociate $\mathrm{CO}$. A similar [C II] $/ \mathrm{H} \alpha$ / young stellar cluster geometry can be found farther along this spiral arm at 13:29:46.9+47:12:29 (J2000) outside the region studied in detail. Interestingly the gas spur S8 itself coincides with $\mathrm{H}$ II regions, lightly enhanced $\mathrm{HI}$, and dust/PAH emission, implying that star formation is still impacting all components of the ISM.

Spur S9-The $\mathrm{H} \alpha$ emission associated with the very bright $\mathrm{H}$ II region at the base of spur S9 shows a bi-conical morphology that is basically oriented perpendicular to the extent of the spur. The peak of the hot dust/PAH emission coincides with the southern $\mathrm{H}$ II region and the location of the very young stellar clusters. However, the hot dust/PAH emission appears to be slightly shifted toward the arm. Given the lower resolution of the IRAC data, we cannot exclude that this is an artifact. The northern H II region within this spur sits in a kink of the molecular gas emission distribution close to the spur's tip. Strong H I emission arises from this region. The [C II] emission peaks in the northern half of this spur, but it is about 25\%-30\% less luminous in surface brightness (or about $50 \%$ in integrated flux) than the emission associated with spur $\mathrm{S} 6$, roughly consistent with the difference in $\mathrm{H} \alpha$ luminosity between the $\mathrm{H}$ II regions of these two spurs.

Given the fact that star formation is concentrated toward the gas spurs, one could conceive that the star formation happening in a spur could trigger more star formation events. We use our spiral arm segment to search for signs of star formation activity triggered by stellar feedback. Given our resolution and tracers used, we find that spur S6 is the only spur showing potential signs of triggered star formation activity among our nine spurs studied-based on its particular morphology.

Spur S6 is not exactly oriented perpendicular to the gas arm, but appears to be tilted more eastward. In addition the low level $\mathrm{CO}$ emission exhibits a hook-like appearance at the tip of the spur. Two young stellar clusters with an age of 3-10 Myr (Chandar et al. 2011, 2016) sit within this hook, while a large number of $\leqslant 3$ Myr young stellar clusters are distributed along the hook continuing along the western side of the spur toward the spiral arm (see Figure 5). Similarly, strong $\mathrm{H} \alpha$ emission is associated with the gas hook and the western side of the gas spur, while more diffuse, low level $\mathrm{H} \alpha$ emission is seen inside the hook. This geometry can suggest that star formation first occurred at the location inside the hook, and that due to stellar feedback onto the surrounding gas, more star formation has been triggered. This could explain part of the tentative age gradient present in this region.

While it is not clear that stellar feedback has indeed triggered star formation within the spurs, it seems clear from the molecular $(\mathrm{CO})$ and ionized $(\mathrm{H} \alpha)$ gas morphology that star formation feedback is impacting the molecular gas distribution (e.g., spurs S4 and S9).

\section{Discussion}

We discuss our results in the context of a simple spiral density wave picture that predicts a clear offset signature between spiral arm and star-forming sites (Section 5.1). Given the surprisingly close relation between gas spurs and starforming sites, we compare our findings to expectations from simulations (Section 5.2).

\subsection{A Simple Spiral Density Wave Picture and Its Implications}

In the framework of a spiral density wave the following picture can be put forward: at the location of the spiral arm potential the gas is efficiently collected, compressed, and starts to collapse and form stars. In this simple picture, one would expect that the collapse of the gas clumps and the subsequent star formation is always taking place in the same location (i.e., at or close to the spiral arm potential). In the case of a spiral density wave, the spiral arm rotates at a constant pattern speed. Thus the differential disk rotation will cause a constantly varying offset between the gas spiral and star-forming regions (that decoupled from the gas motion) due to the difference in age. Note that this picture makes no assumptions about the exact cause of cloud formation or their collapse.

Our results from section Sections 3 and 4 are already qualitatively in disagreement with this picture. However, a certain stochasticity in the star formation onset within GMCs could introduce some scatter. Therefore we measure the deprojected radial offset of different star formation tracers from their neighboring spiral arm location, namely for the $24 \mu \mathrm{m}$ peaks, the individual H II regions and complexes from Lee et al. (2011), and the young ( $<10 \mathrm{Myr})$ stellar clusters (separated into younger and older than $3 \mathrm{Myr}$; Chandar et al. 2016) and compare it to the expected offset for a constant pattern angular speed of $\Omega_{\mathrm{P} \text {, spiral }}=53 \mathrm{~km} \mathrm{~s}^{-1} \mathrm{kpc}^{-1}$ (Querejeta et al. 2016) of a spiral arm with a fixed pitch angle of $i_{\mathrm{p}}=20^{\circ}$ (Patrikeev et al. 2006). In Figure 9 no clear trend in the offset perpendicular from the arm is obvious for stellar clusters younger (older) than 3 Myr. Similarly individual H II regions show a wide spread in that offset, corresponding to times of up to $8 \mathrm{Myr}$ (clearly larger than a $\mathrm{H}$ II region lifetime). A trend for shorter separation times is implied when concentrating on the brightest star-forming regions, as evidenced by the $24 \mu \mathrm{m}$ peaks and the H II complexes; however, no preference for a single separation time is evident. Thus we conclude that our qualitative picture is correct. The varying offset between the young star-forming regions and the gas spiral arm implies that the most massive star formation is not always starting in exactly the same location relative to the spiral arm potential.

Figure 9 paints a complicated picture of star formation in the spiral arm. Most of the young regions nearest to the molecular 


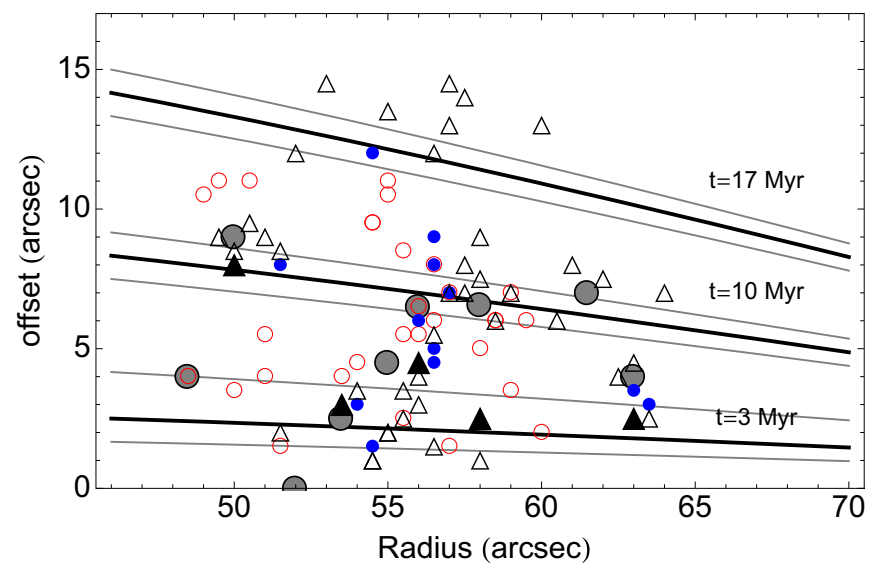

Figure 9. Offset of star-forming regions from a gas spiral arm as a function of the galacto-centric position of the arm. The offset was determined (by eye) in perpendicular direction to the gas arm in a deprojected image; the typical error on both parameters is $\sim 0$. 2 due to the uncertainty in determining the ridge line of the gas arm. Small shifts in radius have been applied for better readability of the plot. Small filled circles show young stellar clusters from Chandar et al. (2016), and the color coding corresponds to young stellar cluster ages of $\log (t$ (year) $)<6.5$ (blue filled) and $6.5 \leqslant \log (t$ (year) $)<7.0$ (red open). The open and filled black triangles mark the locations of individual $\mathrm{H}$ II regions and H II complexes from Lee et al. (2011). $24 \mu \mathrm{m}$ peak positions are given as large gray filled circles. The thick solid lines correspond to the expected offsets from the spiral arm after 3, 10, and $17 \mathrm{Myr}$ ( $\pm 1 \mathrm{Myr})$. The offsets perpendicular to the arm (with a given pitch angle of $i_{\mathrm{p}}=20^{\circ}$; Patrikeev et al. 2006) are based on a simple picture in which star formation occurs instantaneously with the passage of the spiral arm, modeled as a simple kinematic wave with a pattern speed of $\Omega_{\mathrm{P} \text {,spiral }}=53 \mathrm{~km} \mathrm{~s}^{-1} \mathrm{kpc}^{-1}$ (Querejeta et al. 2016) and assuming the rotation curve from Meidt et al. (2013). No clear trend is evident for all or individual star formation tracers.

arm ridge are consistent with forming in the spiral arm, including the embedded star formations sites traced by $24 \mu \mathrm{m}$ and the $\mathrm{H}$ II region complexes. However, the young regions farthest from the arm (including some 3 and $10 \mathrm{Myr}$ old stellar clusters and $\mathrm{H}$ II regions) are beyond where they could be in the case of instantaneous star formation in a simple propagating kinematic wave. These star-forming regions do indeed appear to have formed very near to their present location (i.e., at the location of the spurs). Only if there are positive radial and azimuthal (i.e., outward) flows (on the order of $10-15 \mathrm{~km} \mathrm{~s}^{-1}$ ), the location of these star-forming sites might still be consistent with forming in the spiral arm, as these (additional) radial motions would allow young star formation sites to move much farther away from the spiral ridge than illustrated in Figure 9. However, the presence of such outward radial flows are particularly unlikely. The observed gas kinematics imply strongly radially inward motions already at the location of the gaseous spiral arm (see Meidt et al. 2013).

The star formation sites farthest away from the arm might alternatively arrive at their present location, if their progenitor clouds formed in the spiral arm, but the onset of star formation has been delayed. Adding an additional 5-8 Myr before star formation occurs would allow the $\sim 3$ Myr old stellar clusters and $\mathrm{H}$ II regions to reach their present positions. This timescale is consistent with the crossing time for the observed spiral arm and spur clouds, that is, $t_{\text {cross }}=r_{\text {cloud }} / \Delta v_{\text {cloud }}$ (with $r_{\text {cloud }} \approx$ $40 \mathrm{pc}$ and $\Delta v_{\text {cloud,arm }} \approx 8 \mathrm{~km} \mathrm{~s}^{-1}$ and $\Delta v_{\text {cloud,spur }} \approx 5 \mathrm{~km} \mathrm{~s}^{-1}$ resulting in $t_{\text {cross,arm }} \approx 5 \mathrm{Myr}$ and $t_{\text {cross,spur }} \approx 8 \mathrm{Myr}$ for spiral arm and spur clouds, respectively). At fixed size, low mass clouds with smaller velocity dispersions and longer crossing times would appear farther from the spiral arm ridge than their higher-mass counterparts. Note, however, that not all observed regions (particularly those with smallest offset from the arm) would require such a delay to be consistent with the spiral arm formation scenario.

Alternatively, we can consider a scenario where star formation is initiated within the spiral arm but yields new stars with a delay to explain our observation of star formation occurring within spurs. In this case the delay represents the time to form the spur itself (i.e., from a sheared arm cloud). With the measured offsets it is not possible to distinguish between spur formation through the evolution of spiral arm clouds (e.g., Dobbs 2008; Dobbs \& Pringle 2013) or the case in which the spurs are independently evolving structures forming via gravitational instability, such as envisaged by Kim \& Ostriker (2002). The lack of a clear gradient in the star formation ages across the spurs appears to be inconsistent with other suggested formation mechanisms (e.g., Wada et al. 2011; Renaud et al. 2014; see also the later discussion). The lack of a clear age gradient also disfavors star formation happening solely in the spiral arm (at least in the simple propagating wave picture). We thus conclude that many of the observed star formation sites must be genuinely associated with the spurs, rather than the spiral arm, whereas others are consistent with forming in the spiral arm. More generally, young regions at the observed offsets cannot be ascribed a single formation mechanism or a single characteristic timescale.

From this analysis we can conclude that star formation proceeds with a variety of timescales in or near the spiral arms. Despite the observed complexity in the positions of the sites of recent star formation relative to the spurs, the evidence is consistent with a mixture of mechanisms that lead to both coarse- and fine-tuning of the star formation timescale. Broadly, we identify star formation occurring in two main modes: star formation within the spiral arms and star formation starting independently within spurs, presumably when the clouds created in the process of spur formation are sufficiently massive and bound enough to collapse and form stars. This leads generally to two characteristic zones for observed starforming regions relative to the spiral arm at (roughly) fixed age: near and far from the spiral arm ridge. A spreading throughout these zones is the result of additional fine-tuning in the timescale, determined by properties of the individual clouds themselves. We find that the scatter observed around the arm and spur zones is qualitatively consistent with the additional dependence of the star formation timescale, specifically on the crossing time of the cloud (as discussed previously).

Although the formation of individual spurs and the starforming clouds within them are likely subject to local conditions, we speculate that spur and subsequent star formation depends on gas dynamics on scales larger than molecular clouds and that the process may even arise with material processed independently of the arm cloud population. Our comparison of the $\mathrm{CO}$ distribution imaged at different resolutions suggests that more diffuse molecular gas may be distributed with a regularity reminiscent of the apparent regularity in the spur population. Spur formation in this case might occur through the compression of diffuse gas and subsequent gravitational instability as envisaged by Kim \& Ostriker (2002), rather than as the result of clouds from the arm shearing out as they exit the arm and pass in to the interarm. In the former scenario, a new population of clouds would form as part of the spurs, independently of the spiral arm cloud 
population. More critically, the process of star formation in spurs would be decoupled from star formation in the spiral arms.

The fact that spurs can support star formation independently of star formation starting in the spiral arm would have important implications for global gas consumption within and among galaxies. It has been suggested that in some instances, the gas kinematic characteristic of flow through a spiral arm perturbation may lead to a suppression of star formation due to enhanced turbulence in the spiral shock (Kim \& Ostriker 2002) or as a result of cloud-cloud collisions (Dobbs 2008) or under the influence of dynamical pressure (Meidt et al. 2013). But even when the spiral arm suppresses star formation, the overall dynamics of the spiral can still lead to (at least) modest rates of star formation by promoting spur formation. Such spur-based star formation would then be responsible for the low level of star formation observed in M51 in the region of the spiral arm, where star formation is suppressed (relative to the high rate expected given the observed gas surface density; Meidt et al. 2013), but where spur formation appears to continue successfully. The region with lowered star formation in the spiral under study here directly connects to our segment analyzed at smaller radii. Thus, even when gas kinematics leads to a suppression of star formation internal to the arm, overall spiral arm dynamics could still provide the avenue for star formation through the creation of spurs.

Spur-based star formation would also lead to localized pockets of recent star formation and groupings of young stellar clusters. This can make it difficult to successfully use offsets to measure spiral pattern speeds. A direct implication of our analysis is then that the observed offset between (gas) spiral arms and young star formation has a significant intrinsic scatter. Thus its use to determine the pattern speed of spiral arms (e.g., Egusa et al. 2009) will result in larger uncertainties or less clear answers than naively expected. The significant variation in star formation age and location will also affect other applications that use this simple picture and lead to less clear signatures. This might explain some of the conflicting findings reported in the literature (e.g., Tamburro et al. 2008; Foyle et al. 2010), especially when taking into account that the interpretation of the gas properties are also affected by dissociation and recombination timescales.

\subsection{Formation and Evolution of Gas Spurs}

Theoretical models and simulations (e.g., Kim \& Ostriker 2006, 2002; Dobbs 2008; Dobbs et al. 2011) developed the following picture for the formation of GMCs and subsequent star formation in these dense gas complexes: the formation of gas peaks inside the gas spiral arm can be due to agglomeration of small clouds (Dobbs 2008) and/or self-gravity (Dobbs 2008; Tan 2000; Kim \& Ostriker 2002), or the magneto-Jeans instability (Kim \& Ostriker 2002). These overdensities will become gravitationally unstable and fragment reaching cloud masses up to $10^{6} M_{\odot}$. Due to shear (induced by the spiral potential) these gas fragments are stretched perpendicular to the spiral. Large GMCs can occur at preferred locations within the spiral arms, with a regular spacing given by the Jeans length. Alternatively with the agglomeration scenario there is a quasiperiodic spacing associated with the epicyclic frequency. The gas spurs are the result of these GMC overdensities becoming stretched out.
Our observations support this scenario only partially, as the gas spiral arm appears fairly smooth (with variations within a factor of 2-3 in brightness) and shows no preferred distance between $\mathrm{CO}$ peaks at full $(\sim 40 \mathrm{pc})$ resolution nor the location of the identified GMCs (see Figure 5(a)). However, at a lower resolution of 3 !"0 ( $\sim 110 \mathrm{pc})$ CO peaks with roughly regular spacing become evident. This implies that more diffuse gas on spatial scales larger than typical GMCs $\left(\sim 40 \mathrm{pc} \approx 1^{\prime \prime}\right.$ in M51) is organized in a more regular pattern. As already seen with the feathers (dust lanes emanating from the spiral arm; e.g., La Vigne et al. 2006) the gas spurs closely represent those features seen in simulations. We find no significant difference in the properties of GMCs located in the arm or the spurs, suggesting that no large transformation of more bound structures is happening during the transition from arm to spur. The lower fraction of diffuse emission in the spurs could point to the fact that spurs mark the location of the most efficient compression/ assembly of gas into bound structures. It is interesting to note that the spurs indeed contain GMCs with masses similar to those found by Kim \& Ostriker (2006).

The most interesting observation is that massive clustered star formation seems to be almost entirely associated with gas spurs. This immediately implies that stellar feedback should have a significant impact on the shape and evolution of these gas spurs. Our analysis is inconclusive regarding the location where the onset of star formation occurs. Taking all results together, we find evidence for a star formation onset with no specific preference for a position along a spur and an apparent avoidance of star formation starting within the spiral arm itself. Thus it is not consistent with the simple assumption of a gas density threshold above which star formation starts, as there is no (large) difference in the gas and GMC properties between spiral arm and spur GMCs. The most obvious trend appears to be that the more gas-rich a spur gets, the higher its level of star formation activity is (e.g., S6 and S9), spur S3 being the least gas-rich one, showing no sign of ongoing star formation activity.

Our detailed high-resolution analysis also shows that the use of lower resolution imaging could be misleading, as several star formation events (separated in age) can be present within a single spur and only the brightest event would dominate the light at different spurs. For example, Elmegreen et al. (2014) analyzed $\sim 2^{\prime \prime}$ resolution $3.6 \mu \mathrm{m}, \mathrm{H} \alpha$ and SDSS images to identify the youngest star-forming sites along spiral arms in five nearby spiral galaxies including M51. Their embedded sources 1 and 2 correspond to our spurs S2 and S6. It is clear that star formation has been proceeding in S6 for quite a while (see Section 4.2), including evidence for star formation induced by stellar feedback (see Section 4.3). Therefore it seems that analysis and interpretation need to account for the presence of multiple star formation events or a prolonged period of star formation, even for large complexes. Our derived average GMC masses in spurs S2 and S6 are at the lower $\left(\sim 1 \times 10^{6} M_{\odot}\right)$ and higher $\left(\sim 4 \times 10^{6} M_{\odot}\right)$ end of gas masses observed. However, in any case, they are well below the $10^{7} M_{\odot}$ inferred by Elmegreen et al. (2014) for these regions.

In order to infer an estimate of the star formation efficiency (SFE), we compare the average mass in GMCs to that in young stellar clusters. Note that our estimate for the cluster mass is most likely a lower limit, as stellar clusters already lose a significant fraction of their mass (with a few $10 \%$, depending on the assumptions) within their first $10 \mathrm{Myr}$. We find that spurs $\mathrm{S} 1, \mathrm{~S} 4, \mathrm{~S} 5, \mathrm{~S} 6, \mathrm{~S} 8$, and the arm have an $\mathrm{SFE}<1 \%$, 
while SFE is more than 10 times higher in spurs S2, S7, and S9 (and spur S3 has no stellar clusters and is excluded). These SFEs are low compared to values derived for Galactic GMCs of a few percent and in particular for cloud regions with observed clustered star formation, where SFEs of a few $10 \%$ have been derived (e.g., review by Padoan et al. 2014). The low SFEs could mean that some of the lower mass clouds are not collapsing, resulting in apparently lower SFE, that some of the gas associated with GMCs by the identification algorithm (see the Appendix of Colombo et al. 2014a for details) is not bound, again causing a lower SFE, or that the young stellar clusters have already experienced a much more significant mass loss than assumed. A large population of stellar clusters with masses below our detection limit would result in even lower SFE values, while very young clusters residing in $\mathrm{H}$ II regions could potentially be more massive and lead to potentially higher SFEs. In any case the large variation in derived SFEs suggests that star formation is not proceeding uniformly across the nine spurs considered.

In the turbulent picture one would expect that higher internal turbulence in GMCs leads to higher star formation rates, as more gas can be pushed to higher gas densities suitable for stars to form. Our spur GMCs exhibit slightly lower line widths than arm GMCs, and the arm GMCs have abundant star formation associated with them, whereas no much star formation activity is observed in the arm GMCs - contrary to the simple expectation from the turbulent picture. This supports our interpretation that (massive) star formation in the spiral arm is significantly lowered or not occurring on relevant levels. Meidt et al. (2013) proposed that GMCs in spiral arms might be stabilized through dynamic pressure increased by the streaming motions present in the spiral arms. This scenario could explain simultaneously the lack or shortage of star formation occurring in the spiral arm and the (slightly) lower observed velocity dispersion in the spurs. Other possibilities could be enhanced turbulence in the spiral shock (Kim \& Ostriker 2002) or increased cloud-cloud collisions preventing immediate cloud collapse. In any case, this would imply that spiral galaxies with less strong spiral potential should show a pattern that starts to deviate from M51's strong separation of star formation sites and gas spiral arms, independent of the inferred spiral pattern speed. Thus we interpret the apparent time delay between the spiral arm and the location of star formation as being due to the time it takes to form gravitationally bound structures within GMCs rather than a delay between the presence of such structures and the actual onset of star formation within them.

Recently, Renaud et al. (2014) proposed a different formation mechanism for gas spurs as the one described previously, namely via Kevin-Helmholtz instabilities. The simulated region shown (see Figure 13 of Renaud et al. 2014) roughly resembles the geometry of our region (orientation of the spiral arm with respect to the galaxy center and galactic rotation). The spurs in the simulation have an age gradient in the sense that they start to dissolve at shorter galactic radii while they are still forming at larger galactic radii. In this scenario, one could expect to see an age gradient for starforming sites across neighboring spurs. Our analysis finds no evidence for such an age gradient, implying that the proposed picture is too simplistic or not fully applicable.

In any case, we conclude that there seems to be a close connection between spurs and massive cluster formation in M51, which suggests that spurs might be a requirement for the existence of massive clustered star formation. Thus the mechanism for or cause of gas spur formation is a pre-requisite to form (super-)giant $\mathrm{H}$ II regions and complexes of young stellar clusters. A large statistical sample of arm/spurs GMC and star formation properties is required to properly address cloud and star formation in spurs.

\section{Summary and Conclusion}

In order to better understand the star formation process along spiral arms, we combined high quality and high spatial resolution observations of the ISM and tracers of recent star formation for a spiral arm segment in the disk of the nearby grand-design spiral galaxy M51. The selected arm region is consistent with being driven by a spiral density wave, in the sense that the spiral arm is significantly contributing to the gravitational potential.

Our analysis shows that the picture is more complex than inferred from the simple picture where star formation is started inside a gas spiral arm (by whatever physical process). While a close connection between gas spurs and massive star formation is observed, making a causal connection is difficult. The impact of the recent star formation on the gas spurs is evident in different ISM tracers. In particular, we find that

1. the molecular gas in the selected spiral arm region is distributed into a distinct arm from which gas spurs emanate in an almost perpendicular direction. Detailed analysis shows that GMAs are caused by the blending of gas spurs with their neighboring arm segment and are therefore not single entities (see Section 4.1). While the overall gas surface density in the spurs is lower than in the arm itself, it appears that the gas in the spurs is on average more bound, as the fraction of gas in GMCs is higher in spurs. No other significant differences in properties of GMCs located in the arm or spurs are found.

2. star formation activity is strongly biased toward the spurs, with only a few star-forming sites located inside the spiral arm. No trend in the age of the star formation events is seen either between spurs or along individual spurs. Together with the tendency for massive star formation to occur at a preferred location along spurs, this suggests that the star formation onset is not solely set within or close to the spiral arm. Other stabilizing processes might inhibit the onset of star formation or prolong the collapse of clouds. In addition, rapid dispersal of stars formed in clusters might play a role as well.

3. comparison of the location of emission from heated dust, atomic, and ionized gas reveals that star formation feedback is mostly confined to the region downstream from the spiral arm and often at the tip of the gas spurs. We speculate that the star formation in the upper half of spur S6 is triggered by stellar feedback, given its peculiar shape. Atomic hydrogen emission seems to be due to either $\mathrm{H}_{2}$ dissociation or recombination from the ionized gas. We also identify a region of bright [C II] emission without associated $\mathrm{H} \alpha$ emission but several young stellar clusters, which suggests that [C II] emission is powered over a longer timescale than $\mathrm{H} \alpha$.

4. our detailed analysis suggests that the offset between star formation sites and a gas/dust spiral arm cannot be explained by simple rotation of the spiral arm pattern, as star formation appears not to start at similar locations in 
the spiral arm. Thus interpretations relying on the simple assumption that star formation is started in a single (fixed) location (i.e., the gas arm) can lead to incorrect or inconclusive results. Furthermore this implies that models that predict star formation onset solely in the spiral are too simplistic and need to take into account additional mechanisms that could inhibit or prolong immediate cloud collapse. Possible candidates are the increased dynamic pressure due to streaming motions in the spiral arms, or stabilization due to magnetic fields. We speculate that the offset between star-forming sites and gas arms might be more a function of the strength of the spiral arm potential than the actual pattern spiral speed.

Based on our results we conclude that analysis of a large statistical sample of spurs in galaxies that host differing spiral arm potentials will be required to provide the insights to make significant progress in our understanding of the role of spiral arms for star formation.

We thank the IRAM staff for their support during the observations with the Plateau de Bure interferometer and the $30 \mathrm{~m}$ telescope. S.E.M. and M.Q. acknowledge funding from the Deutsche Forschungsgemeinschaft (DFG) via grant SCHI 536/7-2 as part of the priority program SPP 1573 "ISM-SPP: Physics of the Interstellar Medium." C.L.D. acknowledges funding from the European Research Council for the FP7 ERC starting grant project LOCALSTAR. J.P. acknowledges support from the CNRS programme Physique et Chimie du Milieu Interstellaire (PCMI). M.Q. acknowledges the International Max Planck Research School for Astronomy and Cosmic Physics at the University of Heidelberg (IMPRS-HD). S.G.B. thanks support from Spanish grant AYA2012-32295. We acknowledge financial support to the DAGAL network from the People Programme (Marie Curie Actions) of the European Unions Seventh Framework Programme FP7/2007-2013/ under REA grant agreement number PITN-GA-2011-289313. E.S. thanks NRAO for their support and hospitality during her visits in Socorro. E.S. thanks the Kavli Institute for Theoretical Physics for hospitality during the writing of this paper. IRAM is supported by INSU/CNRS (France), MPG (Germany), and IGN (Spain).

Facilities: IRAM (PdBI), IRAM (30m), HST (ACS), HST (NICMOS), GALEX, NRAO (VLA), Herschel (PACS), Spitzer (IRAC), Spitzer (MIPS).

\section{Appendix}

Tables 5-8 show properties of the individual GMCs, HII regions, and young stellar clusters found in the spurs and spiral arm segment investigated. Further details are provided in the table captions.

Table 5

GMCs Identified in Spurs of the Northern Spiral Segment

\begin{tabular}{|c|c|c|c|c|c|c|}
\hline Spur & ID & $\begin{array}{l}\text { R.A.(J2000) } \\
\quad(\text { deg) }\end{array}$ & $\begin{array}{l}\text { Decl.(J2000) } \\
\quad(\mathrm{deg})\end{array}$ & $\begin{array}{c}r \\
(\mathrm{pc})\end{array}$ & $\begin{array}{c}\Delta v \\
\left(\mathrm{~km} \mathrm{~s}^{-1}\right)\end{array}$ & $\begin{array}{c}M_{\mathrm{H}_{2}} \\
\left(10^{5} M_{\odot}\right)\end{array}$ \\
\hline \multirow[t]{4}{*}{1} & 1291 & 202.4786149 & 47.2077502 & 33 & 4.7 & 9.86 \\
\hline & 1292 & 202.4803521 & 47.2092043 & 44 & 4.9 & 7.93 \\
\hline & 1295 & 202.4792608 & 47.2082393 & 39 & 16.0 & 11.85 \\
\hline & 1296 & 202.4793004 & 47.2088399 & 36 & 5.7 & 14.35 \\
\hline \multirow[t]{3}{*}{2} & 1285 & 202.4778061 & 47.2096689 & 47 & 1.2 & 6.89 \\
\hline & 1286 & 202.4779085 & 47.2102934 & 40 & 7.9 & 19.47 \\
\hline & 1298 & 202.4782993 & 47.2119982 & 26 & 7.8 & 3.26 \\
\hline \multirow[t]{3}{*}{3} & 1344 & 202.4754305 & 47.2108011 & 30 & 9.3 & 3.49 \\
\hline & 1354 & 202.4748221 & 47.2102946 & 61 & 5.8 & 21.17 \\
\hline & 1379 & 202.4749249 & 47.2110108 & 53 & 2.4 & 6.23 \\
\hline 4 & 1356 & 202.4718143 & 47.21131431 & 23 & 4.0 & 61.53 \\
\hline 5 & 1358 & 202.4694169 & 47.2122155 & 60 & 4.4 & 29.90 \\
\hline \multirow[t]{3}{*}{6} & 1348 & 202.4677529 & 47.2119800 & 53 & 10.0 & 44.63 \\
\hline & 1349 & 202.4677036 & 47.2128936 & 75 & 6.8 & 49.56 \\
\hline & 1357 & 202.4668129 & 47.2118519 & 59 & 5.4 & 34.43 \\
\hline \multirow[t]{3}{*}{7} & 1406 & 202.4629751 & 47.2123380 & 38 & 5.7 & 10.05 \\
\hline & 1410 & 202.4624958 & 47.2117164 & 52 & 7.2 & 31.37 \\
\hline & 1419 & 202.4625185 & 47.2113040 & 74 & 1.8 & 35.43 \\
\hline 8 & 1411 & 202.4588562 & 47.21337141 & 25 & 8.2 & 62.20 \\
\hline \multirow[t]{6}{*}{9} & 1399 & 202.4543621 & 47.2109655 & 42 & 5.1 & 10.36 \\
\hline & 1401 & 202.4560851 & 47.2131609 & 16 & 5.0 & 5.45 \\
\hline & 1405 & 202.4558906 & 47.2114785 & 78 & 12.0 & 51.44 \\
\hline & 1412 & 202.4554843 & 47.2134147 & 53 & 1.7 & 7.57 \\
\hline & 1420 & 202.4549731 & 47.2124528 & 45 & 5.4 & 9.28 \\
\hline & 1421 & 202.4546728 & 47.2138573 & 38 & 4.4 & 2.91 \\
\hline
\end{tabular}

Note. The notation of spurs as provided in column (1) is indicated in Figure 3. The remaining columns give the identification number (2), the position (3, 4), the GMC radius (5), the line width (6), and the $\mathrm{H}_{2}$ gas mass (7) derived from the $\mathrm{CO}(1-0)$ luminosity and corrected for He contribution as listed in the GMC catalog of Colombo et al. (2014a). 
Table 6

GMCs Identified in the Arm Segment of the Northern Spiral

\begin{tabular}{|c|c|c|c|c|c|}
\hline ID & $\begin{array}{l}\text { R.A.(J2000) } \\
\quad(\operatorname{deg})\end{array}$ & $\begin{array}{l}\text { Decl.(J2000) } \\
\quad(\mathrm{deg})\end{array}$ & $\begin{array}{c}r \\
(\mathrm{pc})\end{array}$ & $\underset{\left(\mathrm{km} \mathrm{s}^{-1}\right)}{\Delta v}$ & $\begin{array}{c}M_{\mathrm{H}_{2}} \\
\left(10^{5} M_{\odot}\right)\end{array}$ \\
\hline 1288 & 202.4781341 & 47.2064604 & 79 & 7.9 & 89.21 \\
\hline 1290 & 202.4775177 & 47.2075980 & 24 & 18.6 & 33.84 \\
\hline 1294 & 202.4794342 & 47.2058954 & 77 & 8.4 & 26.36 \\
\hline 1301 & 202.4783715 & 47.2070463 & 38 & 5.1 & 7.82 \\
\hline 1307 & 202.4778494 & 47.2064959 & 36 & 6.6 & 5.48 \\
\hline 1340 & 202.4724277 & 47.2092676 & 0 & 4.2 & 0.98 \\
\hline 1346 & 202.4720983 & 47.2101305 & 59 & 11.5 & 32.37 \\
\hline 1347 & 202.4657128 & 47.2105135 & 35 & 4.9 & 12.34 \\
\hline 1352 & 202.4754194 & 47.2094228 & 72 & 6.9 & 29.01 \\
\hline 1353 & 202.4737470 & 47.2096134 & 73 & 5.8 & 29.44 \\
\hline 1355 & 202.4705114 & 47.2108802 & 28 & 4.6 & 1.43 \\
\hline 1365 & 202.4769357 & 47.2077326 & 62 & 6.1 & 16.35 \\
\hline 1367 & 202.4750843 & 47.2089325 & 48 & 1.8 & 8.57 \\
\hline 1368 & 202.4699346 & 47.2103279 & 62 & 12.5 & 49.36 \\
\hline 1375 & 202.4761133 & 47.2084144 & 44 & 9.2 & 15.29 \\
\hline 1377 & 202.4682569 & 47.21079201 & 11 & 7.2 & 79.60 \\
\hline 1378 & 202.4661434 & 47.2108190 & 25 & 11.3 & 35.56 \\
\hline 1381 & 202.4764315 & 47.2069278 & 62 & 6.4 & 33.85 \\
\hline 1388 & 202.4725072 & 47.2090741 & 42 & 6.7 & 5.24 \\
\hline 1400 & 202.4633815 & 47.2105062 & 22 & 2.2 & 1.93 \\
\hline 1409 & 202.4564362 & 47.2109857 & 48 & 6.7 & 19.54 \\
\hline 1417 & 202.4554359 & 47.2094391 & 69 & 12.1 & 28.28 \\
\hline 1426 & 202.4599843 & 47.2110239 & 39 & 5.1 & 15.02 \\
\hline 1432 & 202.4561624 & 47.2101547 & 38 & 9.6 & 13.58 \\
\hline 1433 & 202.4609935 & 47.2107303 & 64 & 7.5 & 30.85 \\
\hline 1442 & 202.4549386 & 47.2098358 & 36 & 4.5 & 4.00 \\
\hline 1448 & 202.4618436 & 47.2103964 & 39 & 1.8 & 5.30 \\
\hline
\end{tabular}

Note. The columns give the identification number (1), the position $(2,3)$, the GMC radius (4), the line width (5), and the $\mathrm{H}_{2}$ gas mass (6) derived from the $\mathrm{CO}(1-0)$ luminosity and corrected for He contribution as listed in the GMC catalog of Colombo et al. (2014a).
Table 7

H II Regions Identified in Northern Spiral Segment

\begin{tabular}{|c|c|c|c|c|c|}
\hline Region & ID & $\begin{array}{l}\text { R.A.(J2000) } \\
\text { (deg) }\end{array}$ & $\begin{array}{l}\text { Decl.(J2000) } \\
\quad(\mathrm{deg})\end{array}$ & $\begin{array}{c}\log \left(L_{\mathrm{H} \alpha}\right) \\
\left(\log \left(\mathrm{erg} \mathrm{s}^{-1}\right)\right)\end{array}$ & $\begin{array}{c}r \\
(\mathrm{pc})\end{array}$ \\
\hline \multirow[t]{3}{*}{1} & 10901 & 202.4788818 & 47.208076 & 37.475 & 44.22 \\
\hline & 11070 & 202.4794006 & 47.208359 & 37.195 & 35.74 \\
\hline & 11086 & 202.4794159 & 47.208946 & 37.198 & 23.58 \\
\hline \multirow[t]{4}{*}{2} & 10690 & 202.4779358 & 47.209621 & 37.142 & 30.58 \\
\hline & 10450 & 202.4778290 & 47.209972 & 38.519 & 104.64 \\
\hline & 10932 & 202.4788055 & 47.210018 & 37.218 & 22.48 \\
\hline & 10816 & 202.4784851 & 47.211365 & 37.585 & 41.64 \\
\hline \multirow[t]{2}{*}{4} & 8291 & 202.4715424 & 47.211002 & 39.181 & 162.49 \\
\hline & 8786 & 202.4718170 & 47.211468 & 37.431 & 42.37 \\
\hline \multirow[t]{2}{*}{5} & 7881 & 202.4695282 & 47.212265 & 37.218 & 30.21 \\
\hline & 8100 & 202.4700165 & 47.212067 & 37.427 & 39.79 \\
\hline 6 & 6795 & 202.4669800 & 47.212589 & 39.471 & 188.28 \\
\hline \multirow[t]{3}{*}{7} & 5732 & 202.4627228 & 47.211338 & 37.695 & 40.16 \\
\hline & 5902 & 202.4635162 & 47.212296 & 37.572 & 47.16 \\
\hline & 5841 & 202.4631805 & 47.212852 & 38.354 & 71.11 \\
\hline \multirow[t]{3}{*}{8} & 4690 & 202.4594727 & 47.212654 & 37.637 & 46.43 \\
\hline & 4540 & 202.4587860 & 47.213089 & 37.413 & 32.79 \\
\hline & 4554 & 202.4589386 & 47.214386 & 37.566 & 39.43 \\
\hline \multirow[t]{3}{*}{9} & 4088 & 202.4557800 & 47.211254 & 39.101 & 129.70 \\
\hline & 4121 & 202.4561462 & 47.212753 & 37.850 & 55.27 \\
\hline & 4010 & 202.4547729 & 47.211903 & 37.349 & 30.95 \\
\hline \multirow[t]{5}{*}{ arm } & 10634 & 202.4779205 & 47.206657 & 37.326 & 33.16 \\
\hline & 10755 & 202.4781647 & 47.207058 & 37.399 & 31.69 \\
\hline & 9797 & 202.4753571 & 47.209400 & 37.476 & 40.53 \\
\hline & 7346 & 202.4682922 & 47.210773 & 37.943 & 42.74 \\
\hline & 8213 & 202.4701996 & 47.210915 & 37.147 & 27.27 \\
\hline
\end{tabular}

Note. The notation of spurs as provided in column (1) is indicated in Figure 3. The remaining columns give the identification number $(2)$, the position $(3,4)$, the logarithm of the $\mathrm{H} \alpha$ luminosity $L_{\mathrm{H} \alpha}(5)$, and the $\mathrm{H}$ II region radius (6) from the $\mathrm{H}$ II region group catalog of Lee et al. (2011). Values are derived assuming a distance to M51a of $7.6 \mathrm{Mpc}$, and the properties of the $\mathrm{H}$ II regions have been corrected correspondingly.

Table 8

Stellar Clusters Identified in Northern Spiral Segment

\begin{tabular}{|c|c|c|c|c|c|}
\hline Region & ID & $\begin{array}{l}\text { R.A.(J2000) } \\
\quad(\mathrm{deg})\end{array}$ & $\begin{array}{l}\text { Decl.(J2000) } \\
\quad(\mathrm{deg})\end{array}$ & $\begin{array}{c}\log (t) \\
(\log (\text { year }))\end{array}$ & $\begin{array}{c}M_{\star} \\
\left(10^{3} M_{\odot}\right)\end{array}$ \\
\hline \multirow[t]{3}{*}{1} & 149411 & 202.4790344 & 47.2080193 & 6.56 & 7.0 \\
\hline & 150175 & 202.4795380 & 47.2084045 & 6.78 & 6.4 \\
\hline & 153018 & 202.4797974 & 47.2097244 & 6.88 & 1.6 \\
\hline \multirow[t]{5}{*}{2} & 155581 & 202.4789276 & 47.2108917 & 6.84 & 21.5 \\
\hline & 153350 & 202.4769440 & 47.2098770 & 6.84 & 39.0 \\
\hline & 152222 & 202.4772186 & 47.2093582 & 6.90 & 1.8 \\
\hline & 154051 & 202.4785461 & 47.2101517 & 6.44 & 4.7 \\
\hline & 154498 & 202.4782562 & 47.2103729 & 6.00 & 9.2 \\
\hline \multirow[t]{5}{*}{4} & 155911 & 202.4720154 & 47.2110329 & 6.38 & 18.7 \\
\hline & 155822 & 202.4718170 & 47.2109871 & 6.58 & 51.7 \\
\hline & 155199 & 202.4712219 & 47.2107048 & 6.20 & 18.5 \\
\hline & 157826 & 202.4706268 & 47.2119026 & 6.78 & 1.3 \\
\hline & 156475 & 202.4728851 & 47.2112885 & 6.78 & 1.3 \\
\hline 5 & 158075 & 202.4695892 & 47.2120247 & 6.56 & 3.4 \\
\hline \multirow[t]{2}{*}{6} & 157786 & 202.4667053 & 47.2118950 & 6.00 & 52.3 \\
\hline & 158632 & 202.4673309 & 47.2122879 & 6.38 & 22.2 \\
\hline
\end{tabular}


Table 8

(Continued)

$\left.\begin{array}{lcccc}\hline \hline \text { Region } & \text { ID } & \begin{array}{c}\text { R.A.(J2000) } \\ (\mathrm{deg})\end{array} & \begin{array}{c}\text { Decl.(J2000) } \\ (\mathrm{deg})\end{array} & \begin{array}{c}\log (t) \\ (\log (\text { year }))\end{array} \\ \hline & 159154 & 202.4674835 & 47.2125168 & 6.46 \\ \left(10^{3} M_{\odot}\right)\end{array}\right)$

Note. The notation of spurs as provided in column (1) is indicated in Figure 3. The remaining columns give the identification number (2), the position (3, 4), the logarithm of the age (5), and the stellar mass (6) of the young $(t \leqslant 10 \mathrm{Myr}$ ) stellar clusters in the catalog of Chandar et al. (2016). Values are derived assuming a distance to M51a of $7.6 \mathrm{Mpc}$, and the properties of the stellar clusters have been corrected correspondingly.

\section{References}

Bastian, N., Gieles, M., Efremov, Y. N., \& Lamers, H. J. G. L. M. 2005, A\&A, 443, 79

Calzetti, D., Chandar, R., Lee, J. C., et al. 2010, ApJL, 719, L158

Chandar, R., Whitmore, B. C., Calzetti, D., et al. 2011, ApJ, 727, 88

Chandar, R., Whitmore, B. C., Dinino, D., et al. 2016, ApJ, 824, 71

Ciardullo, R., Feldmeier, J. J., Jacoby, G. H., et al. 2002, ApJ, 577, 31

Colombo, D., Hughes, A., Schinnerer, E., et al. 2014a, ApJ, 784, 3

Colombo, D., Meidt, S. E., Schinnerer, E., et al. 2014b, ApJ, 784, 4

Corder, S., Sheth, K., Scoville, N. Z., et al. 2008, ApJ, 689, 148

Dobbs, C. L. 2008, MNRAS, 391, 844

Dobbs, C. L., Burkert, A., \& Pringle, J. E. 2011, MNRAS, 413, 2935

Dobbs, C. L., \& Pringle, J. E. 2013, MNRAS, 432, 653

Dumas, G., Schinnerer, E., Tabatabaei, F. S., et al. 2011, AJ, 141, 41

Egusa, F., Koda, J., \& Scoville, N. 2011, ApJ, 726, 85

Egusa, F., Kohno, K., Sofue, Y., Nakanishi, H., \& Komugi, S. 2009, ApJ, 697, 1870

Elmegreen, B. G., Seiden, P. E., \& Elmegreen, D. M. 1989, ApJ, 343, 602

Elmegreen, D. M. 1980, ApJ, 242, 528

Elmegreen, D. M., Elmegreen, B. G., Erroz-Ferrer, S., et al. 2014, ApJ, 780,32

Foyle, K., Rix, H.-W., Walter, F., \& Leroy, A. K. 2010, ApJ, 725, 534

Gutiérrez, L., Beckman, J. E., \& Buenrostro, V. 2011, AJ, 141, 113

Kennicutt, R. C., Jr, Armus, L., Bendo, G., et al. 2003, PASP, 115, 928

Kim, W. T., \& Ostriker, E. C. 2002, ApJ, 570, 132

Kim, W. T., \& Ostriker, E. C. 2006, ApJ, 646, 213

Koda, J., Scoville, N., Sawada, T., et al. 2009, ApJL, 700, L132

La Vigne, M. A., Vogel, S. N., \& Ostriker, E. C. 2006, ApJ, 650, 818

Lee, J. H., Hwang, N., \& Lee, M. G. 2011, ApJ, 735, 75

Meidt, S. E., Rand, R. J., Merrifield, M. R., Shetty, R., \& Vogel, S. N. 2008 , ApJ, 688, 224
Meidt, S. E., Schinnerer, E., García-Burillo, S., et al. 2013, ApJ, 779, 45 Meidt, S. E., Schinnerer, E., Knapen, J. H., et al. 2012, ApJ, 744, 17 Mentuch Cooper, E., Wilson, C. D., Foyle, K., et al. 2012, ApJ, 755, 165 Mutchler, M., Beckwith, S. V. W., Bond, H., et al. 2005, BAAS, 37, 452 Padoan, P., Federrath, C., Chabrier, G., et al. 2014, in Protostars and Planets VI, ed. H. Beuther et al. (Tucson, AZ: Univ. Arizona Press), 77

Parkin, T. J., Wilson, C. D., Schirm, M. R. P., et al. 2013, ApJ, 776, 65

Patrikeev, I., Fletcher, A., Stepanov, R., et al. 2006, A\&A, 458, 441 Pety, J., Schinnerer, E., Leroy, A. K., et al. 2013, ApJ, 779, 43

Querejeta, M., Meidt, S. E., Schinnerer, E., et al. 2015, ApJS, 219, 5

Querejeta, M., Meidt, S. E., Schinnerer, E., et al. 2016, A\&A, 588, A33

Rand, R. J., \& Kulkarni, S. R. 1990, ApJL, 349, L43

Renaud, F., Bournaud, F., Emsellem, E., Elmegreen, B., \& Teyssier, R. 2014,

in ASP Conf. Ser. 480, Structure and Dynamics of Disk Galaxies, ed.

M. S. Seigar \& P. Treuthardt (San Fransisco, CA: ASP), 247

Rosolowsky, E., \& Leroy, A. 2006, PASP, 118, 590

Schinnerer, E., Meidt, S. E., Pety, J., et al. 2013, ApJ, 779, 42

Schuster, K. F., Kramer, C., Hitschfeld, M., Garcia-Burillo, S., \& Mookerjea, B. 2007, A\&A, 461, 143

Scoville, N. Z., Polletta, M., Ewald, S., et al. 2001, AJ, 122, 3017

Sheth, K., Regan, M., Hinz, J. L., et al. 2010, PASP, 122, 1397

Shetty, R., \& Ostriker, E. C. 2006, ApJ, 647, 997

Tamburro, D., Rix, H.-W., Walter, F., et al. 2008, AJ, 136, 2872

Tan, J. C. 2000, ApJ, 536, 173

Tilanus, R. P. J., \& Allen, R. J. 1989, ApJL, 339, L57

Tully, R. B. 1974, ApJS, 27, 449

Vogel, S. N., Kulkarni, S. R., \& Scoville, N. Z. 1988, Natur, 334, 402

Vogel, S. N., Rand, R. J., Gruendl, R. A., \& Teuben, P. J. 1993, PASP, 105,666

Wada, K., Baba, J., \& Saitoh, T. R. 2011, ApJ, 735, 1

Walter, F., Brinks, E., de Blok, W. J. G., et al. 2008, AJ, 136, 2563

Whitmore, B. C., Chandar, R., Kim, H., et al. 2011, ApJ, 729, 78 